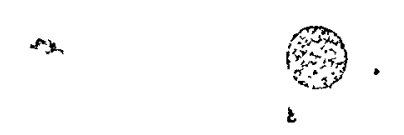

$\angle A-S U B-99-10$

\title{
Investigation and Prediction of RF Window Performance in APT Accelerators
}

\author{
Final Report \\ Los Alamos National Laboratory BOA0409J004-3P, Task 43
}

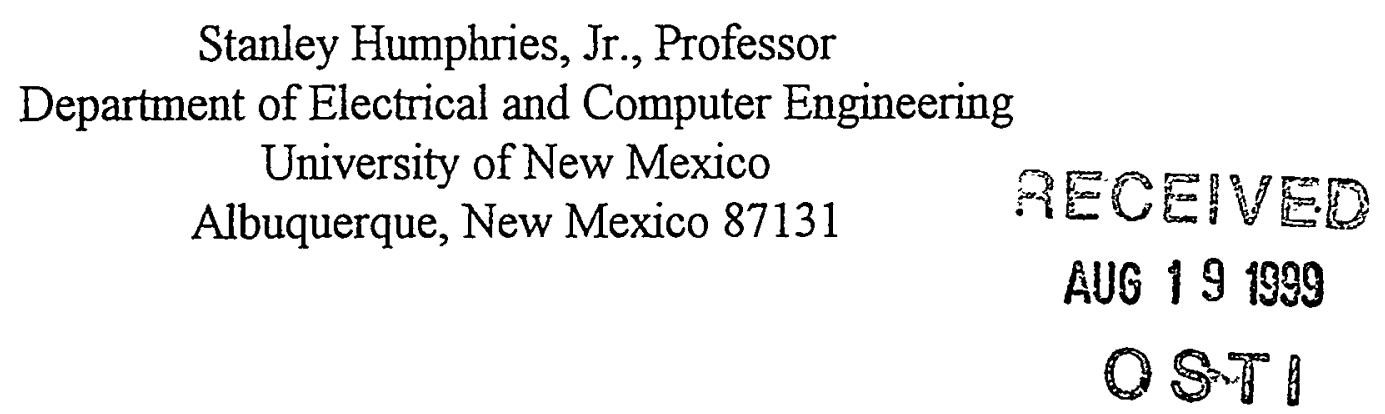

The work described in this report was performed between November 1996 and May 1997 in support of the APT (Accelerator Production of Tritium) Program at Los Alamos National Laboratory. The goal was to write and to test computer programs for charged particle orbits in $\mathrm{RF}$ fields. The well-documented programs were written in portable form and compiled for standard personal computers for easy distribution to LANL researchers. They will be used in several APT applications, including the following.

- Minimization of multipactor effects in the moderate $\beta$ superconducting linac cavities under design for the APT accelerator.

- Investigation of supression techniques for electron multipactoring in high-power RF feedthroughs.

- Modeling of the response of electron detectors for the protection of high power RF vacuum windows.

In the contract period two new codes, Trak_RF and WaveSim, were completed and several critical benchmark tests were carried out. Trak_RF numerically tracks charged particle orbits in combined electrostatic, magnetostatic and electromagnetic fields. WaveSim determines frequencydomain RF field solutions and provides a key input to Trak_RF. The two-dimensional programs handle planar or cylindrical geometries. They have several unique characteristics. 


\section{DISCLAIMER}

This report was prepared as an account of work sponsored by an agency of the United States Government. Neither the United States Government nor any agency thereof, nor any of their employees, make any warranty, express or implied, or assumes any legal liability or responsibility for the accuracy, completeness, or usefulness of any information, apparatus, product, or process disclosed, or represents that its use would not infringe privately owned rights. Reference herein to any specific commercial product, process, or service by trade name, trademark, manufacturer, or otherwise does not necessarily constitute or imply its endorsement, recommendation, or favoring by the United States Government or any agency thereof. The views and opinions of authors expressed herein do not necessarily state or reflect those of the United States Government or any agency thereof. 


\section{DISCLAIMER}

Portions of this document may be illegible in electronic image products. Images are produced from the best available original document. 


\section{Trak RF}

- Finite-element field calculations on a conformal triangular mesh with variable resolution.

- Capacity to include planar or cylindrical electrostatic, magnetostatic and RF field solutions on independent meshes with arbitrary translations and rotations

- Option for orbit information from other programs or automatic generation of particle distributions

- Energy-dependent secondary emission coefficients for up to 10 different materials.

- Flexible orbit stopping conditions.

The WaveSim mesh and solution methods are similar to those of Superfish. The program gives accurate field solutions along complex material boundaries. In comparison with Superfish, the program has several advanced capabilities to address the full range of APT applications.

\section{WaveSim}

- Handles up to 400,000 elements and 127 regions of linear dielectric or ferromagnetic materials.

- Includes effects of material losses.

- Derives scattering solutions in open structures as well as resonant solutions.

Work under this contract has been well-documented during the contract with progress reports and publications. The remainder of this report consists of the following papers that describe code development and benchmark multipactor calculations.

1. S. Humphries, Jr., TRAK_RF Benchmark Tests - CERN Coaxial Feed, Progress Report 1, March, 1997, unpublished.

2. S. Humphries, Jr. and D. Rees, Trak_RF - Simulation of Electromagnetic Fields and Particle Trajectories in High-power $R \bar{F}$ Devices, Proc. 1997 Applied Computational Electromagnetics Conference, Monterrey, CA, March 1997, to be published. 
3. S. Humphries ${ }_{2} \mathrm{Jr}$., WaveSim - Frequency-domain Finite-element Electromagnetic Field Solutions, March 1997, unpublished.

4. S. Humphries, Jr., Multipactor Calculations for the LANL APT Superconducting Linac Cavities, Progress Report 2, April, 1997, unpublished.

5. S. Humphries, Jr. and D. Rees, Electron Multipactor Code for High-power RF Devices, Proc. 1997 Particle Accelerator Conference, Vancouver, BC, May 1997, to be published. 


\title{
TRAK_RF - Simulation of Electromagnetic Fields and Particle Trajectories in High-power RF Devices
}

\author{
Stanley Humphries, Jr. \\ Department of Electrical and Computer Engineering \\ University of New Mexico \\ Albuquerque, New Mexico 87131
}

\author{
and \\ Daniel Rees \\ ATP Program \\ Los Alamos National Laboratory \\ Los Alamos, New Mexico 87545
}

\begin{abstract}
A. Introduction
Trak_RF is an integrated finite-element software system to model charged particle trajectories in combined electrostatic, magnetostatic and electromagnetic fields. The program is an extension of the Trak 3.0 gun design code ${ }^{1}$. New capabilities include frequency-domain calculations of electromagnetic fields in resonant cavities and open space and particle tracking in time-dependent fields. A unique feature is the ability to combine up to three independent numerical solutions for static electric and magnetic fields and RF modes. The current version is limited to two-dimensional static fields (planar or cylindrical geometries) and three-dimensional RF fields in two-dimensional structures. The latter case includes propagating modes in waveguides and modes of type $\mathrm{TE}_{\mathrm{mnp}}$ or $\mathrm{TM}_{\mathrm{mnp}}$ in cylindrical resonators.

The program has been developed to investigate contributions of multipactoring to breakdowns on $\mathrm{RF}$ windows in high-power accelerators. A cooperative program on Accelerator Production of Tritium to maintain the US nuclear weapons stockpile has recently been initiated at Los Alamos National Laboratory (LANL) and the Savannah River Site ${ }^{2}$. The goal is a continuously operating proton linac to generate a $1.3 \mathrm{GeV}$ beam with an average current of $100 \mathrm{~mA}$ (130 MW beam power). The accelerator demands powerful RF systems with high reliability. A critical area of concern is the possibility of breakdowns on RF vacuum windows that must transmit MW power levels. To address the issue, an experimental and theoretical program of window testing and development has been initiated at LANL ${ }^{3}$. Starting in October 1996, the University of New Mexico has supported this effort through the development of particle and radiation diagnostics to warn of impending breakdowns and computer codes to help understand the role of stray electrons in window failure.
\end{abstract}


This paper reviews the status of the Trak_RF program. This computational tool has been completed and benchmark tested. We plan extensive studies of multipactoring and advanced window designs through Spring of 1997. Section B describes the conformal triangular meshes used in the program and the range of quasi-static field solutions that can be incorporated in orbit calculations. Section $\mathrm{C}$ reviews features of the finite-element methods used for frequency-domain RF solutions. The solver handles open and closed systems with frequency-dependent wall and material losses. Section D covers the particle tracking capabilities. The program can handle electron multiplication resulting from secondary emission with energy-dependent emission coefficients. Finally, Section E describes a benchmark test to demonstrate features of the code. The calculation follows electron orbits in a 700 $\mathrm{kW}$ coaxial feedthrough with a static magnetic mirror field to repel electrons from an alumina window.

\section{B. Conformal meshes and static field computations.}

All static and dynamic field calculations in Trak_RF are carried out on conformal meshes. As an example, Fig. 1 shows a mesh of triangular elements for the calculation of TEM waves in a highpower coaxial feedthrough. The advantage is apparent - the edges of elements conform closely to curved and angled material boundaries. As a result, each element can be uniquely associated with a material region. This is an important feature in a particle tracking code because it allows an accurate identification of particles striking material surfaces. Section D discusses some of the benefits of the finite element formulation for $\mathrm{RF}$ calculations. In static field solutions, there are three major advantages compared to finite-difference calculations: 1) the finite element method gives accurate field values near metal surfaces, 2) the technique represents field discontinuities at the boundaries of dielectrics and ferromagnetic materials, and 3 ) it is easy to implement Neumann conditions on angled and curved boundaries.

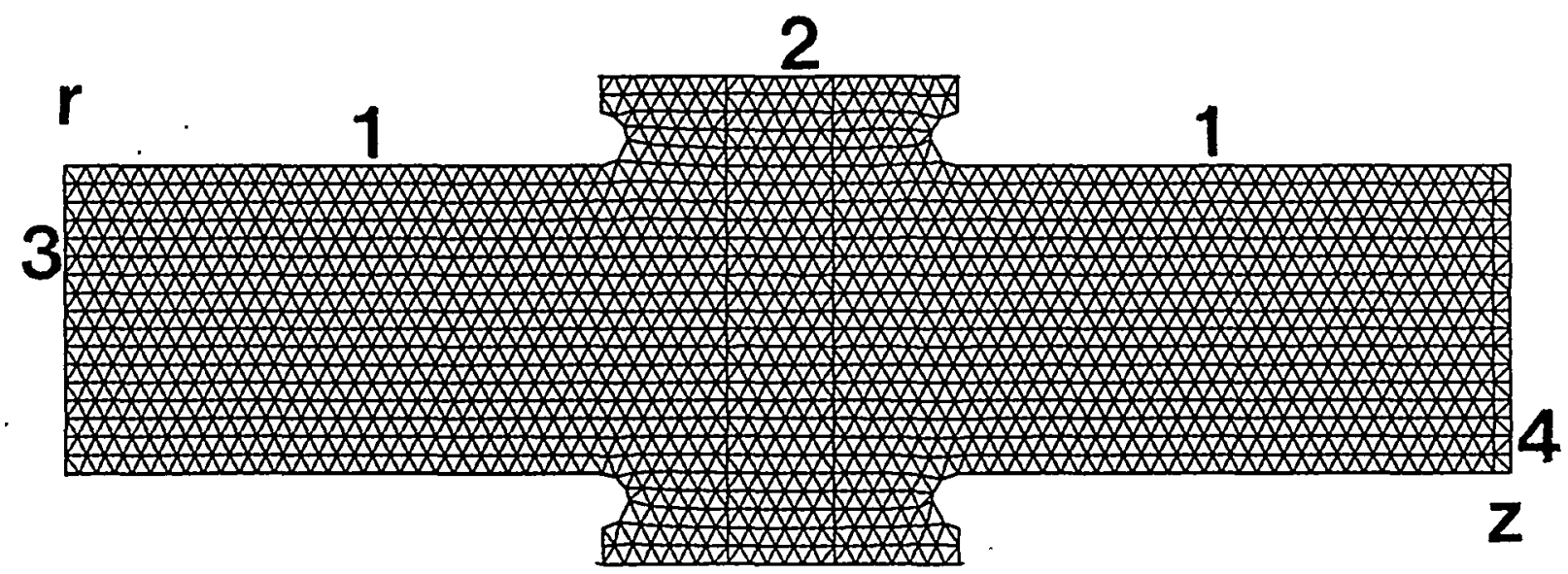

Figure 1. Conformal triangular mesh - TEM waves $\left(H_{\mathrm{e}}\right)$ in a high-power coaxial feedthrough. $r_{\min }: 0.8^{\prime \prime}, r_{\max }: 3.3^{\prime \prime}, z_{\min }:-4.0^{\prime \prime}, z_{\max }: 4.0^{\prime \prime}$. Region 1: Vacuum with Neumann boundaries (metal wall). Region 2: Aluminum insulator. Region 3: Drive boundary $\left(0^{\circ}\right.$ phase). Region 4: Resistive termination layer. 
TrakRF uses the standard mesh gen J Jr and static field solvers of the TriComp system [5]. This suite of finiteelement programs runs on IBM-standard personal computers. Boundary information is entered through an interactive drafting program or from drafting like AutoCAD. The basic electrostatic and magnetostatic solvers use the linear finite-element formulation described in Refs. [6] and [7] with solutions by successive over-relaxation. They analyze files of boundary and material information to produce output files of vertex coordinates and corresponding values of electrostatic or vector potential.

\section{RF FIELD COMPUTATIONS}

The derivation of finite-element equations for twodimensional frequency-domain $R F$ fields is reviewed in Ref. [7]. As an example, consider the equations for a planar structure with no variation in $z$ with electric field polarization $E_{z}$. The associated differential equation is

$$
-\nabla \times\left(\frac{1}{\mu} \nabla \times E\right)=-\epsilon \omega^{2} E+j \omega J_{0},
$$

where $\omega$ is the angular frequency of the radiation. The quantities $\mu$ and $\epsilon$ may have may have complex values to represent losses from resistivity or non-ideal materials. The current source $J_{0}$ contains information on the amplitude and phase of drive regions. The finite-element equations for wave propagation at the test vertices are derived from area integrals of Eq. 1 over surrounding elements and vertices. The result is

$$
\begin{gathered}
E_{x o}\left(-\sum_{t} W_{t}+\omega^{2} \sum_{t} \frac{\epsilon a_{t}}{3}\right)+\sum_{t} E_{x t}= \\
j \omega \sum_{t} \frac{J a_{t}}{3} .
\end{gathered}
$$

In general, the quantities $E_{z i}$ are complex numbers to represent amplitude and phase. The index $i$ refers to the vertices and elements surrounding a test vertex marked $o$. The quantities $\epsilon_{i}, \mu_{i}$ and $J_{i}$ are the material properties and current density of the elements. Expressions for the geometric coefficients $W_{1}$ are given in Ref. [7]. Equation 2 represents a large set of coupled linear equations, one for each mesh vertex. The set is solved in TrakRF using sparse matrix inversion methods. The complex values of $E_{z i}$ give the physical electric field at a given RF phase. Numerical derivatives give the magnetic field components $B_{x}$ and $B_{y}$.

A significant advantage of the finite-element method is the ability to define ideal absorbing layers of arbitrary shape to represent free-space boundary conditions. The procedure is to set up a thin layer of width $\delta$ on the outside of the solution volume. The imaginary part of the dielectric constant in the layer is ass ed the value $\epsilon^{\prime \prime}=-\sigma / \omega$, where the conductivity is matched to the impedance of the adjacent medium,

$$
\sqrt{\frac{\mu}{\epsilon}}=\frac{1}{\sigma \delta} .
$$

The performance of absorbing layers equals or exceeds that of look-back techniques [8]. The advantages are that termination layers can have any shape or orientation and do not place restrictions on the time step in time-domain solutions.

Figure 2 shows an example of a resonant calculation, the $\pi$ mode of the LANL five-cell superconducting cavity array for proton beams with $\beta=0.64$. The outer radius is $19.4 \mathrm{~cm}$ and there is a $20 \mathrm{~cm}$ beam pipe on the left that is not shown. A small capacitive probe (bottom-right) drives the mode. The program searches for the zero crossing of the imaginary part of the response of a sensor near the outer radius. The predicted frequency of 701.6 MHZ is in good agreement with SuperFish and MAFIA results.

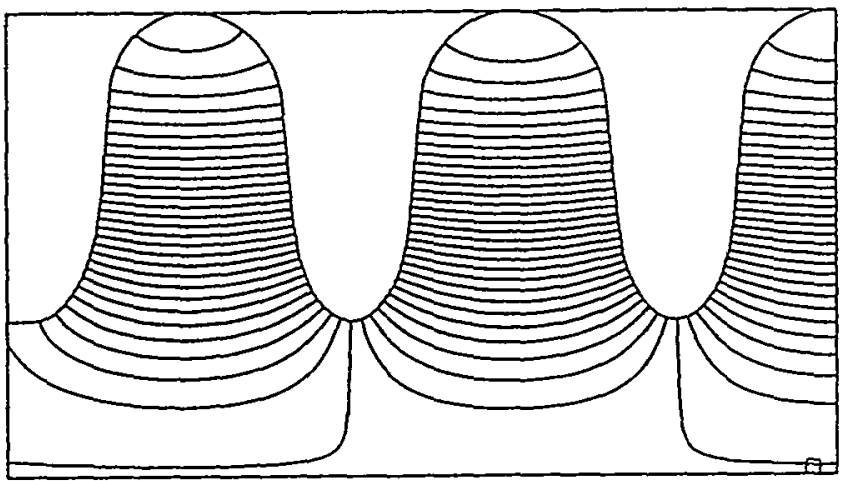

Figure 2. Electric field lines $-\pi$ mode of the LANL $\beta=$ 0.645 -cell superconducting cavity array. $f=701.6 \mathrm{MHZ}$.

\section{CHARGED-PARTICLE ORBIT CALCULATIONS}

Charged-particle orbit calculations in TrakRF are straightforward. They involve Runge-Kutta integrations using numerically-calculated field components. The main challenge is organization of the broad range of possibilities. The program can handle three numerical field solutions on independent conformal meshes: electrostatic, magnetostatic, and electromagnetic. The motivation for this versatility is to model stray electron control near RF windows using sweeping fields. Field geometries can be mixed in any combination. The static solutions may have either rectangular geometry (variations in $\mathrm{x}$ and $\mathrm{y}$ with infinite extent in $z$ ) or cylindrical (variations in $r$ and $z$ with azimuthal symmetry). There are four possibilities for electromagnetic fields: rectangular geometries with primary field components $\mathrm{E}_{\mathrm{z}}$ or $\mathrm{H}_{\mathrm{z}}$ or cylindrical systems with solutions for $\mathrm{E}_{\theta}$ or $\mathrm{H}_{\theta}$. TrakRF uses a reference 

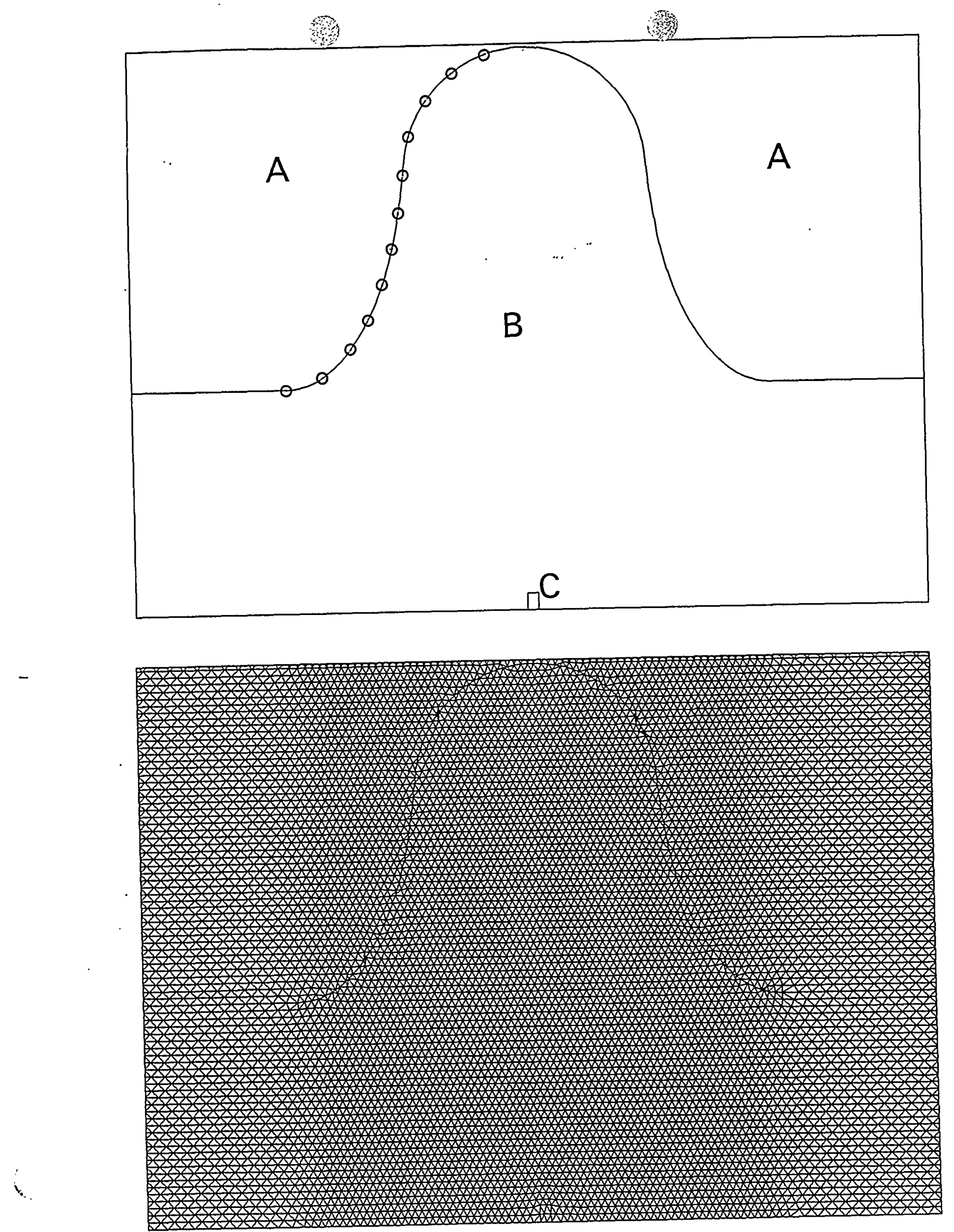


\section{TRAK RF Benchmark Tests CERN Coaxial Feed}

\section{Stanley Humphries, Jr. Department of Electrical and Computer Engineering University of New Mexico Albuquerque, New Mexico $\mathbf{8 7 1 3 1}$ March 1997}

\section{A. Introduction}

Trak $R^{1}$ is an advanced multipactor code developed for RF design work in the APT (Accelerator Production of Tritium) Program ${ }^{2}$ at Los Alamos National Laboratory. It can handle two-dimensional finite-element solutions for RF, electrostatic and magnetostatic fields on independent conformal triangular meshes. The RF solutions are quite general compared to codes like Superfish ${ }^{3}$. The WaveSim program directly calculates either standing or traveling wave solutions with the option for lossy materials. The particle tracking routines have been extensively tested in the Field Precision Trak electron gun design program ${ }^{4}$. Several new features have been added to support secondary emission from materials. Trak_RF can handle up to five different emitting materials with user-supplied curves for the energy-dependent secondary emission coefficient.

This report summarizes results from a benchmark test suggested by B. Rusnak and F. Krawczyk of LANL. The goal was to replicate experiments and simulations carried out at CERN on a high power superconducting coaxial feed. The tests were performed with a standing wave in a coaxial resonator with properties listed in Table 1. Figure $1 a$ shows the measured response of an electron collector as a function of power in the line. Figure $1 b$ shows results of the CERN simulation.

\begin{tabular}{|l|l|}
\hline \multicolumn{2}{|c|}{ Table 1 } \\
\multicolumn{2}{|c|}{ Coaxial Feed Parameters } \\
\hline Inner radius & $0.01475 \mathrm{~m}$ \\
\hline Outer radius & $0.05150 \mathrm{~m}$ \\
\hline Impedance & $75 \Omega$ \\
\hline Frequency & $352 \mathrm{MHZ}$ \\
\hline Wavelength & $0.8532 \mathrm{~m}$ \\
\hline
\end{tabular}


CERN COAX SW
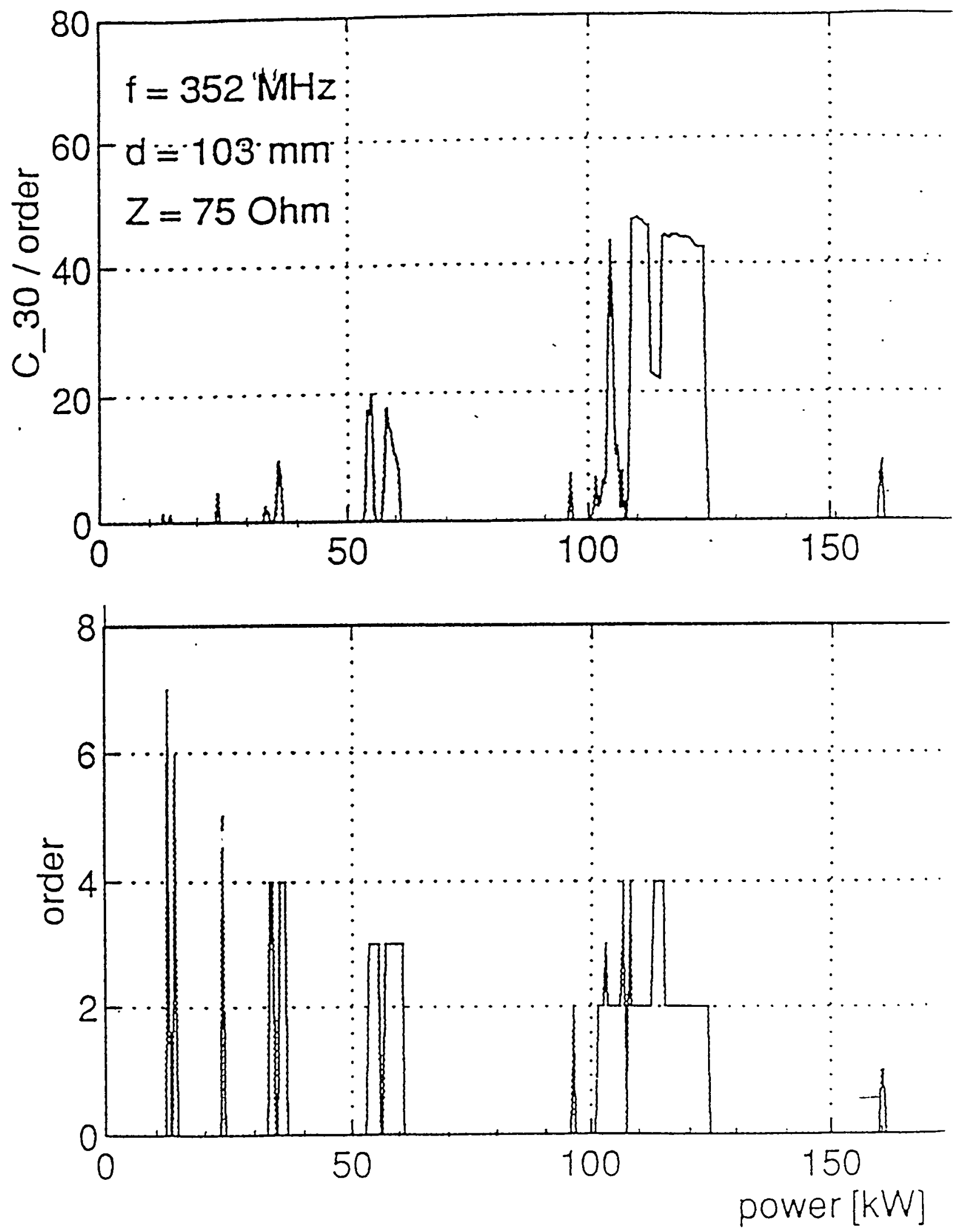

Figure 1. CERN multipactor studies in a coaxial feed (supplied by B. Rusnak). a) Experimental measurements of electron flux as a function of line power. b) Simulation predictions. 


\section{B. Secondary Emission Coefficients}

The secondary emission coefficient $\delta$ for electrons normally incident on clean metals generally exceeds unity over a range from $100 \mathrm{eV}$ to $1 \mathrm{keV}$. A extensive compendium of data from the CRC Handbook ${ }^{5}$ is reproduced as Figure 2. The table lists four items: the peak value of $\delta$ and the corresponding energy, and the energy range where $\delta \geq 1$. I developed a spreadsheet template to fit a smooth curve to these parameters to create tabular input for Trak_RF. Figure 3 shows the curve for Niobium. My curve differed from that used in the CERN simulations (Figure 4) where the multiplication region extended to $1600 \mathrm{eV}$ and there appeared to be no low-energy cutoff.

\section{RF Field Calculation}

For the RF solution I set up an ideal TEM wave propagating along a coaxial waveguide with inner and outer radii of $0.01475 \mathrm{~m}$ and $0.05150 \mathrm{~m}$. Figure 5 shows the solution region and contours of $\mathrm{rH}_{\theta}$ at $\mathrm{t}=0.0$. The contours are parallel to electric field lines. The wave was generated by a specified drive field condition on the left-hand boundary at $\mathrm{z}=-0.1 \mathrm{~m}$ with a phase of $-42^{\circ}$. This gave the field variation

$$
r H_{\theta}=0.8218 \cos (2 \pi f t)
$$

at $\mathrm{z}=0.0$, the position of electron emission. This value corresponds to a peak current $\mathrm{I}_{0}=2 \pi \mathrm{rH}_{\theta}$ $=5.164 \mathrm{~A}$. The power carried by the traveling wave is therefore $\mathrm{P}=\mathrm{Z}_{0} \mathrm{I}_{0}{ }^{2} / 2=1 \mathrm{~kW}$. The electric field amplitude at the outer radius is $E_{\mathrm{ro}}=6021 \mathrm{~V} / \mathrm{m}$. Given the baseline, electron orbits could be investigated at different power levels by changing the field scaling factor.

The main solution region is a homogenous, loss less medium with $\epsilon=\epsilon_{\mathrm{o}}$ and $\mu=\mu_{\mathrm{o}}$. The right-hand boundary in Fig. 5. is a matched termination layer to absorb the traveling wave. For a layer thickness of $0.005 \mathrm{~m}$, a complex dielectric constant

$$
\epsilon=\epsilon_{o}(1.0-27.08 j),
$$

gives a match to the characteristic impedance of free space $(377.3 \Omega)^{6}$. We can verify complete absorption (equivalent to zero Standing-Wave-Ratio) by checking that the amplitudes of complex field quantities are constant over the length of the solution.

The dark regions at the inner and outer radii are layers that represent secondary emission surfaces. In the RF solution, the single layer of elements in the layers are assigned void properties $\left(\epsilon=\infty, \mu=\mu_{o}\right)$ so that they act as open circuit boundaries, the equivalent of a metal 
Figure 2. Electron secork ary emission coefficients, reproducec om the Handbook of Chemistry and Physics (CRC Press, Boca Raton, 1994).

\section{SECONDARY ELECTRON EMISSION}

The secondary emission yield, or secondary emission ratio, $\delta$, is the average number of secondary electrons emitted from a bombarded material for every incident primary electron. It is a function of the primary electron energy $E_{p}$. The maximum yield $\delta_{\text {max }}$ corresponds to a primary electron energy $E_{\text {pmax }}$ (see figure). The two primary electron energies corresponding to a yield of unity are denoted the furst and second crossovers ( $E_{l}$ and $\left.E_{n}\right)$. An insulating target, or a conducting target that is clectrically floating, will charge positively or negatively depending on the primary electron energy. For $E_{1}<E_{\mathrm{p}}<E_{11}, \delta>1$ and the surface charges positively provided there is a collector present that is positive with respect to the target. For $E_{\mathrm{p}}<E_{1}$ or $E_{\mathrm{p}}$ $>E_{11} . \delta<1$, and the surface charges negatively with respect to the potential of the source of primary electrons.

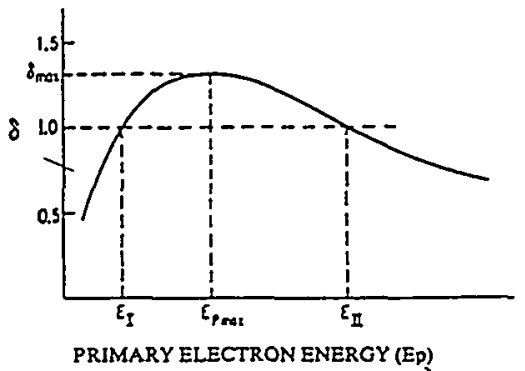

The secondary emission yield is very sensitive to surface contamination, such as oxide films and carbon deposits. Whenever possible, yields believed to be most typical of clean surfaces have been selected. The yields are for measurements at room temperature and normal incidence of the primary electrons.

\begin{tabular}{|c|c|c|c|c|c|c|c|c|c|}
\hline Element & $\delta_{\max }$ & $\begin{array}{c}E_{\mathrm{pmax}} \\
(\mathrm{eV})\end{array}$ & $\begin{array}{c}E_{1} \\
(\mathrm{eV})\end{array}$ & $\begin{array}{c}E_{\mathrm{HI}} \\
(\mathrm{eV})\end{array}$ & Element & $\delta_{\max }$ & $\begin{array}{c}E_{\mathrm{pmax}} \\
(\mathrm{eV})\end{array}$ & $\begin{array}{c}E_{\mathrm{I}} \\
(\mathrm{eV})\end{array}$ & $\begin{array}{c}E_{1 \mathrm{t}} \\
(\mathrm{eV})\end{array}$ \\
\hline$A g$ & 1.5 & 800 & 200 & $>2000$ & $\mathrm{Li}$ & 0.5 & 85 & None & None \\
\hline AI & 1.0 & 300 & 300 & 300 & $\mathrm{Mg}$ & 0.95 & 300 & None & None \\
\hline Au & 1.4 & 800 & 150 & $>2000$ & Mo & 1.25 & 375 & 150 & 1200 \\
\hline B & 1.2 & 150 & 50 & 600 & $\mathrm{Na}$ & 0.82 & 300 & None & None \\
\hline $\mathrm{Ba}$ & 0.8 & 400 & None & None & $\cdot \mathrm{Nb}$ & 1.2 & 375 & 150 & 1050 \\
\hline $\mathrm{Bi}$ & 1.2 & 550 & None & None & $\mathrm{Ni}$ & 1.3 & 550 & 150 & $>1500$ \\
\hline $\mathrm{Be}$ & 0.5 & 200 & Nöne & None & $\mathrm{Pb}$ & 1.1 & 500 & 250 & 1000 \\
\hline$C$ (diamond) & 2.8 & 750 & None & $>5000$ & $\mathrm{Pd}$ & $>1.3$ & $>250$ & 120 & None \\
\hline$C$ (graphite) & 1.0 & 300 & 300 & 300 & $\mathrm{Pt}$ & 1.8 & 700 & 350 & 3000 \\
\hline$C$ (soot) & 0.45 & 500 & None & None & $\mathbf{R b}$ & 0.9 & 350 & None & None \\
\hline Cd & 1.1 & 450 & 300 & 700 & $\mathrm{Sb}$ & 1.3 & 600 & 250 & 2000 \\
\hline $\mathrm{Co}$ & 1.2 & 600 & 200 & None & $\mathrm{Si}$ & 1.1 & 250 & 125 & 500 \\
\hline Cs & 0.7 & 400 & None & None & $\mathrm{Sn}$ & 1.35 & 500 & None & None \\
\hline $\mathrm{Cu}$ & 1.3 & 600 & 200 & 1500 & $\mathrm{Ta}$ & 1.3 & 600 & 250 & $>2000$ \\
\hline $\mathrm{Fe}$ & 1.3 & 400 & 120 & 1400 & $T h$ & 1.1 & 800 & None & None \\
\hline $\mathrm{Ga}$ & 1.55 & 500 & 75 & None & $\mathrm{Ii}$ & 0.9 & 280 & None & None \\
\hline $\mathrm{Ge}$ & 1.15 & 500 & 150 & 900 & $\mathrm{Tl}$ & 1.7 & 650 & 70 & $>1500$ \\
\hline $\mathrm{Hg}$ & 1.3 & 600 & 350 & $>1200$ & $w$ & 1.4 & 650 & 250 & $>1500$ \\
\hline$K$ & 0.7 & 200 & None & None & Zr & 1.1 & 350 & None & None \\
\hline
\end{tabular}

\footnotetext{
Compound

Alkali halides

$\mathrm{CsCl}$

$\mathrm{KBr}$ (erystal)

$\mathrm{KCl}$ (crystal)

$\mathrm{KCl}$ (layer)

KI (crystal)

KI (layer)

LiF (crystal)

LiF (layer)

$\mathrm{NaBr}$ (crystal)

$\mathrm{NaBr}$ (layer)

$\mathrm{NaCl}$ (crystal)

$\mathrm{NaCl}$ (layer)

$\mathrm{NaF}$ (crystal)

$\mathrm{NaF}$ (layer)

NaI (crystal)

NaI (layer)

$\mathrm{RbCl}$ (layer)

Oxides

$\mathrm{Ag}_{2} \mathrm{O}$

$\mathrm{Al}_{2} \mathrm{O}_{3}$ (layer)

$\mathrm{BaO}$ (layer)

$\mathrm{BeO}$
}

$\delta_{\max }$

$\begin{array}{r}\delta_{\text {max }} \\ 6.5 \\ 14 \\ 12 \\ 7.5 \\ 10 \\ 5.6 \\ 8.5 \\ 5.6 \\ 24 \\ 6.3 \\ 14 \\ 6.8 \\ 14 \\ 5.7 \\ 19 \\ 5.5 \\ 5.8 \\ 1.0 \\ 2-9 \\ \hline 2.3-4.8 \\ 3.4 \\ \hline\end{array}$

Sulfides

$\mathrm{MoS}_{2}$

$\mathrm{PbS}$

$\mathrm{WS}_{2}$

$\mathrm{ZnS}$

Others

$\mathrm{BaF}_{2}$ (layer)

$\mathrm{CaF}_{2}$ (layer)

$\mathrm{BiCs}_{3}$

BiCs

$\mathrm{GeCs}$.

$\mathrm{Rb}_{3} \mathrm{Sb}$

$\mathrm{SbCs}_{3}$

Mica

Glasses

$\begin{array}{rr}2.2 & 500 \\ 1.2 & 400 \\ 20-25 & 1500 \\ 3-15 & 400-1500 \\ 1.2 & \\ 2.1-4 & 400 \\ 3.2 & 640 \\ 1.1 & \\ 1.2 & \\ 1.0 & 500 \\ 1.8 & \\ & \\ 4.5 & \\ 3.2 & \\ 6 & \\ 1.9 & 1000 \\ 7 & 1000 \\ 7.1 & 700 \\ 6 & 450 \\ 2.4 & 700 \\ 2-3 & 350 \\ & 450\end{array}$




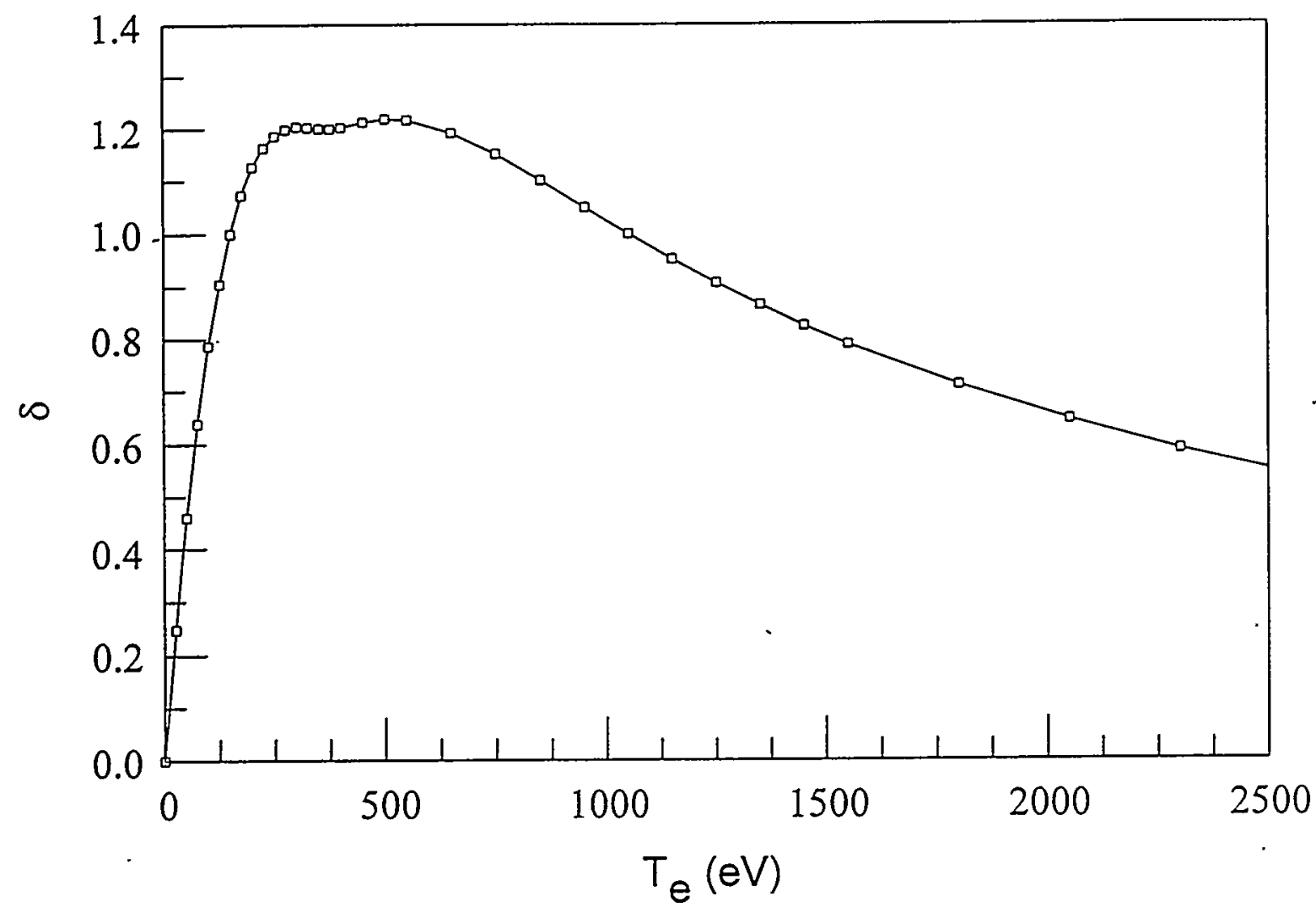

Figure 3. Secondary electron emission coefficient for Niobium as a function of incident electron energy. Input to Trak_RF program derived from the parameters of Fig. 2.

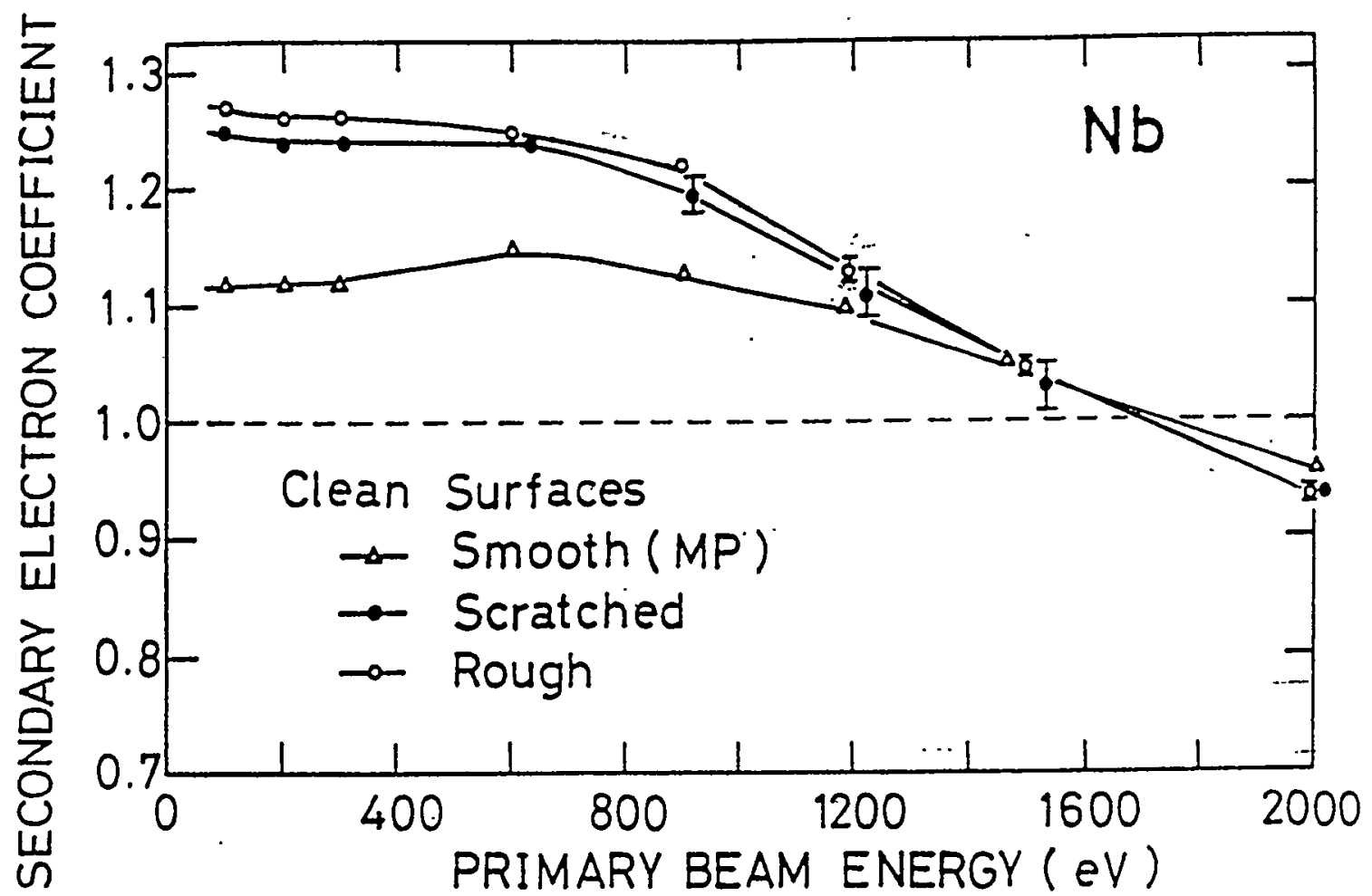

Figure 4. Secondary electron emission coefficient for Niobium as a function of incident electron energy used for the CERN study. 


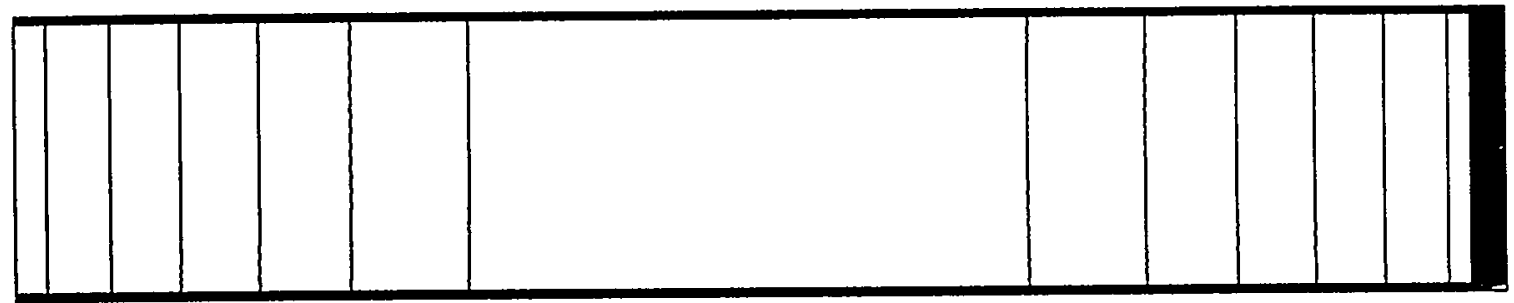

Figure 5. WaveSim RF field solution, ideal TEM wave in a coaxial waveguide - contours of $\mathrm{rH}_{\theta} \cdot \mathrm{r}_{\min }: 0.01375 \mathrm{~m}, \mathrm{r}_{\max }: 0.5250 \mathrm{~m}, \mathrm{z}_{\min }:-0.1000 \mathrm{~m}, \mathrm{z}_{\max }: 0.1050 \mathrm{~m}$. Region 1: Solution volume, vacuum. Region 2: Absorbing termination layer. Region 3: Inner secondary emission surface. Region 4: Outer secondary emission surface. Region 5: Drive boundary. Region 6: Short-circuit boundary.

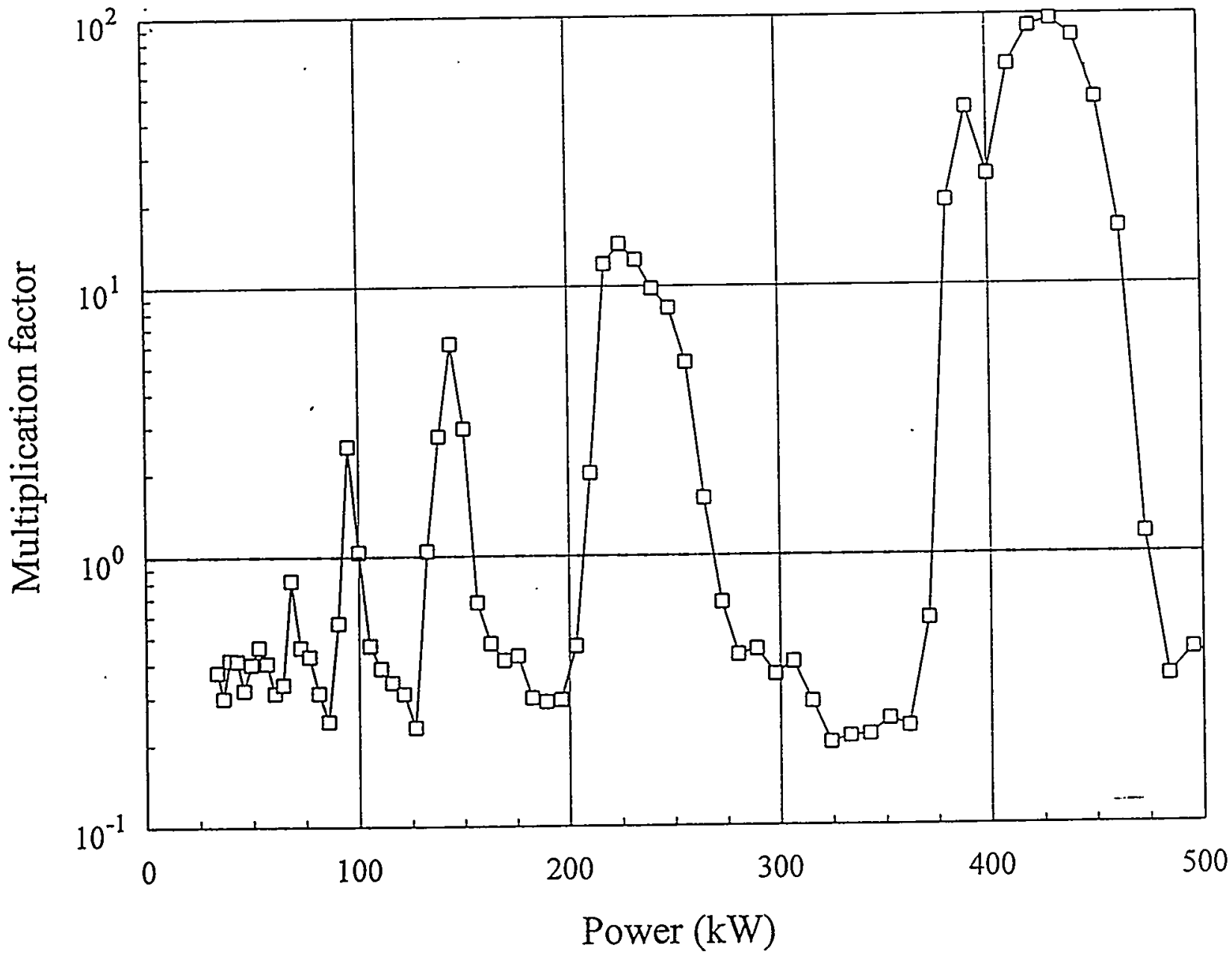

Figure 6. Electron multiplication factor as a function of traveling wave power in the $75 \Omega$ transmission line. Electron emission from the outer boundary. 
surface for the field component $\mathrm{rH}_{\theta}$. During orbit tracking electrons entering these regions are assumed to have struck a secondary emission surface. Appendices 1 and 2 show the input files for mesh generation and control of the RF solution.

\section{Electron Multiplication Factors}

The multipactor simulations used the RF solution and followed 181 electron orbits initiated on the outer wall of the waveguide at $\mathrm{z}=0.0 \mathrm{~m}$. The orbits covered the accelerating phase of the wave $\left(-90.0^{\circ}\right.$ to $\left.90.0^{\circ}\right)$ in $1^{\circ}$ intervals. Each electron started with a multiplication factor of unity. When an electron entered a secondary emission element, the following actions were taken.

- The multiplication factor was multiplied by the quantity $\delta\left(T_{e}\right)$ where Te is the electron kinetic energy at the material surface.

- A flag was checked to see if the electron was in a secondary region on the previous time step.

A set flag or a value of $T_{e}$ than a cutoff value $(5 \mathrm{eV})$ indicate that the electron hit the surface during the decelerating phase of the RF electric field. In this case, the orbit was terminated. Orbits were also stopped if the multiplication factor dropped below $10^{-4}$.

- To continue the orbit, the electron was moved to its position before entering the material and assigned zero kinetic energy.

- Program statistics were updated.

Tracking continued until the orbit terminated or exceeded a maximum elapsed time. All runs of this report used a maximum run time of $150 \mathrm{~ns}$, equal to about $53 \mathrm{RF}$ periods. A global multiplication factor (ratio of the number of final to initial electrons) was calculated after tracing all model electron orbits. A factor greater than unity was an indication of a multipactor band. The program also gives information on total surface hits organized by Region. In the present problem these numbers indicated the relative importance of collisions on the inner and outer electrodes.

Figure 6 shows a plot of the multiplication factor as a function of power for electrons emitted on the outer surface of the CERN waveguide. Multipactor bands are evident over the power range 0.0 to $500 \mathrm{~kW}$. The electron distribution resulted from single electrode effects - all hits were on the outer conductor. Figure 7 shows the multiplication factor resolved according to emission phase at $220 \mathrm{~kW}$. The emission phase range for electron multiplication is consistent with the analytic planar gap theory ${ }^{7}$. I also made a series of runs over the power range with electron emission from the inner boundary. There were about 300 hits on the inner boundary relatively independent of power level. The number of hits on the outer boundary was considerably larger in the multipactor bands. Apparently, electron strikes on the inner boundary did not lead to multiplication. Electrons that crossed the gap to strike the outer boundary initiated 


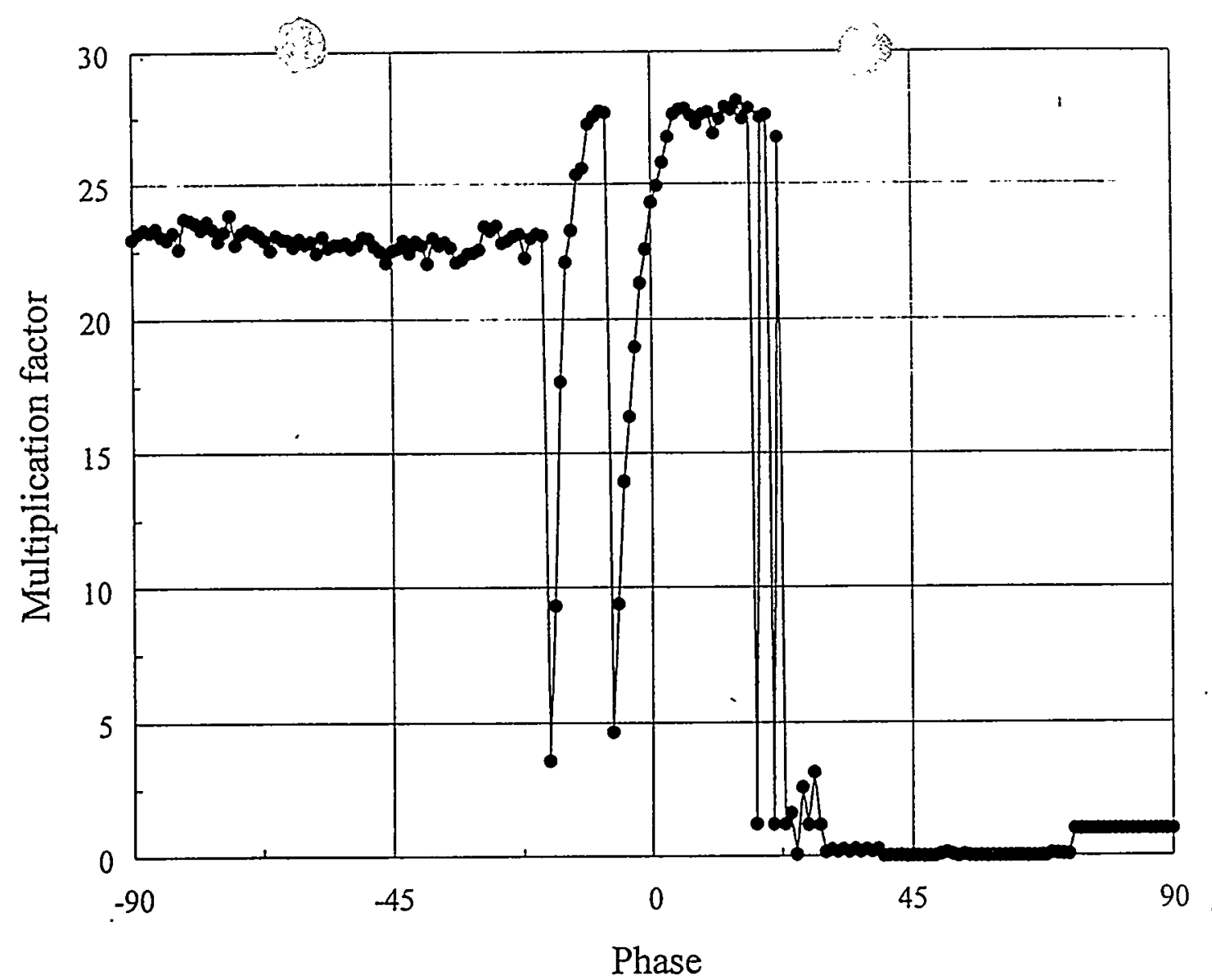

Figure 7. Electron multiplication factor as a function of emission phase for a $220 \mathrm{~kW}$ traveling wave in the $75 \Omega$ line.

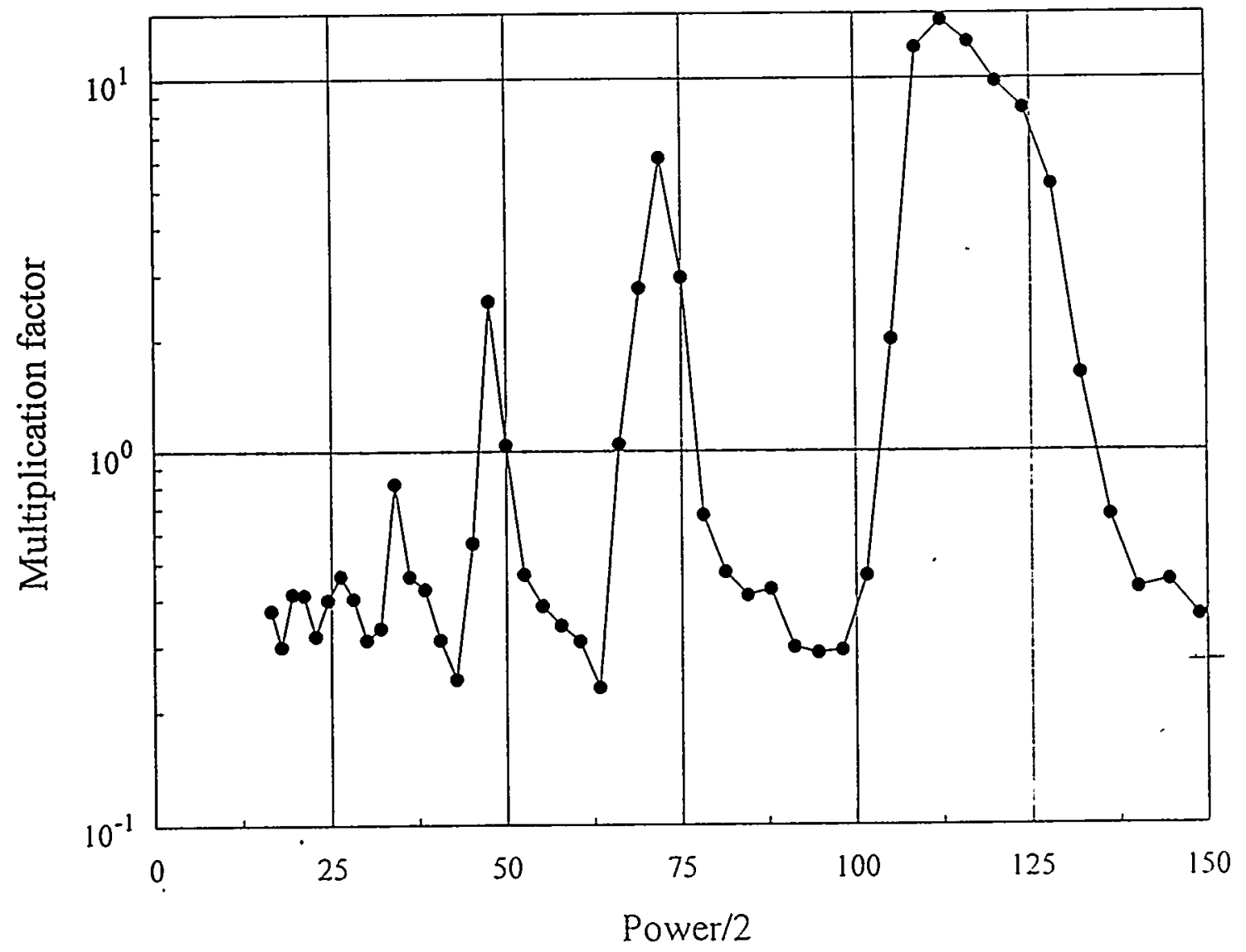

Figure 8. Electron multiplication factor as a function of traveling wave power in the $75 \Omega$ transmission line. Data of Fig. 6 plotted for comparison to Fig. 1. 
secondaries that followed the behavior of the outer wall simulations. In consequence, the band structure was quite similar to that of Fig. 6.

A comparison of Fig. 6 to Fig. 1 shows that the Trak_RF results are similar to the CERN results except for a factor of 2 difference on the power axis. For comparison, Fig. 8 shows a plot of the multiplication factor as function of power divided by two in the lower power regime. It is possible that my definition of power differs from those of the CERN standing wave experiments. Another difference in the Trak-RF simulations is that multipactoring falls into broad bands without the extensive microstructure of the CERN results. I am at a loss to explain the physical basis of the small scale resonances in Fig. 1.

\section{E. Additional Features of the Multipactor Solutions}

Trak_RF has an option to record detailed statistics on secondary emission events. For example, Table $\overline{2}$ shows the collision history for an electron initiated on the outer conductor at a phase of $-60^{\circ}$ for a $225 \mathrm{~kW}$ line power. The table lists the following electron parameters at collisions: axial position, kinetic energy, interpolated secondary emission coefficient, and radial electric field. Within the $150 \mathrm{~ns}$ time limit the electron struck the outer wall 17 times for a total multiplication factor of 18.42 . The data confirms the validity of the $\delta\left(T_{e}\right)$ interpolations and shows that the electric field was either positive or at a negative-to-positive crossing point. The axial position illustrates an interesting effect. We expect no first order axial motion for a free electron oscillating in an RF field because the ponderomotive force is quite small. On the other hand, the phasing of forces in the traveling wave produces a net axial translation because the electron velocity reverts to zero with each collisions. The axial position shown in Table 2 shifts at approximately constant velocity a distance $0.011 \mathrm{~m}$ in $53 \mathrm{RF}$ cycles.

To conclude, Fig. 9 plots the radial motion of an electron emitted at phase $-90.0^{\circ}$ for the 225 $\mathrm{kW}$ solution. Collisions with the outer wall appear at the top. The electron moves a significant distance across the gap. Collisions are regularly spaced every three RF periods, indicating 3rd order multipactoring.

\section{References}

1. S. Humphries, Jr. and D. Rees, Trak_RF - Simulation of Particle Trajectories in High-power $R F$ Devices, Proc. ACES Workshop (Monterrey, 1997), to be published.

2. G. Lawrence, et.al., Conventional and Superconducting RF Linac Design for the APT Project, Proc. 1996 Linear Acc. Conf (Geneva, 1996), to be published.

3. K. Halbach and R.F. Holsinger, Particle Accelerators 7, 213 (1976).

4. S. Humphries, Jr., J. Comp. Phys. 125, 488 (1996).

5. Handbook of Chemistry and Physics (CRC Press, Boca Raton, 1994), 12-107.

6. S. Humphries, Jr., Field Solutions on Computers (CRC Press, Boca Raton, 1997), Chap. 13.

7. See, for instance, S. Humphries, Jr., Principles of Charged Particle Acceleration (John

Wiley, New York, 1986), Sect. 14.5. 
TABLE 2

\section{Parameters: Collisions with Secondary Emission Regions}

\begin{tabular}{|c|c|c|c|}
\hline$z(t)$ & $\mathrm{Te}$ & Delta & Er \\
\hline 1. $58526 \mathrm{E}-03$ & $3.11684 E+02$ & $1.20428 \mathrm{E}+00$ & $7.85539 \mathrm{E}+04$ \\
\hline $3.02211 E-03$ & $2.81945 \mathrm{E}+02$ & $1.20119 \mathrm{E}+00$ & $5.71750 \mathrm{E}+04$ \\
\hline $3.09174 E-03$ & $2.15390 \mathrm{E}+02$ & $1.15067 \mathrm{E}+00$ & $8.48804 E+04$ \\
\hline $2.39152 E-03$ & $3.23422 \mathrm{E}+02$ & $1.20324 \mathrm{E}+00$ & $-2.81579 E+03$ \\
\hline $2.79988 \mathrm{E}-03$ & $5.71297 \mathrm{E}+02$ & 1. $21402 \mathrm{E}+00$ & $4.18221 \mathrm{E}+04$ \\
\hline $2.56989 \mathrm{E}-03$ & $4.03624 E+02$ & $1.20355 \mathrm{E}+00$ & $6.60904 E+04$ \\
\hline $2.79752 \mathrm{E}-03$ & $1.73197 \mathrm{E}+02$ & $1.06847 \mathrm{E}+00$ & $8.53647 E+04$ \\
\hline $3.06924 \mathrm{E}-03$ & $3.14265 \mathrm{E}+02$ & $1.20416 \mathrm{E}+00$ & $-1.11285 E+04$ \\
\hline $4.02560 \mathrm{E}-03$ & $5.49371 \mathrm{E}+02$ & $1.21707 \mathrm{E}+00$ & $4.72221 E+04$ \\
\hline $4.24531 E-03$ & $3.21789 \mathrm{E}+02$ & $1.20346 \mathrm{E}+00$ & $7.65299 \mathrm{E}+04$ \\
\hline $4.14202 E-03$ & $2.66367 \mathrm{E}+02$ & $1.19564 \mathrm{E}+00$ & $6.36553 E+04$ \\
\hline $5.50282 E-03$ & $1.89540 E+02$ & $1.10626 \mathrm{E}+00$ & $8.56588 \mathrm{E}+04$ \\
\hline $6.50312 E-03$ & $3.15803 E+02$ & $1.20407 \mathrm{E}+00$ & $-6.68718 E+03$ \\
\hline $8.69001 E-03$ & $5.95937 E+02$ & $1.20899 \mathrm{E}+00$ & $3.65689 \mathrm{E}+04$ \\
\hline $9.46903 E-03$ & $4.61756 \mathrm{E}+02$ & $1.21477 \mathrm{E}+00$ & $5.71180 \mathrm{E}+04$ \\
\hline $9.90244 \mathrm{E}-03$ & $2.80697 E+02$ & $1.20084 \mathrm{E}+00$ & $7.79166 \mathrm{E}+04$ \\
\hline $1.09362 E-02$ & $2.65786 \mathrm{E}+02$ & $1.19537 \mathrm{E}+00$ & $6.11006 \mathrm{E}+04$ \\
\hline
\end{tabular}




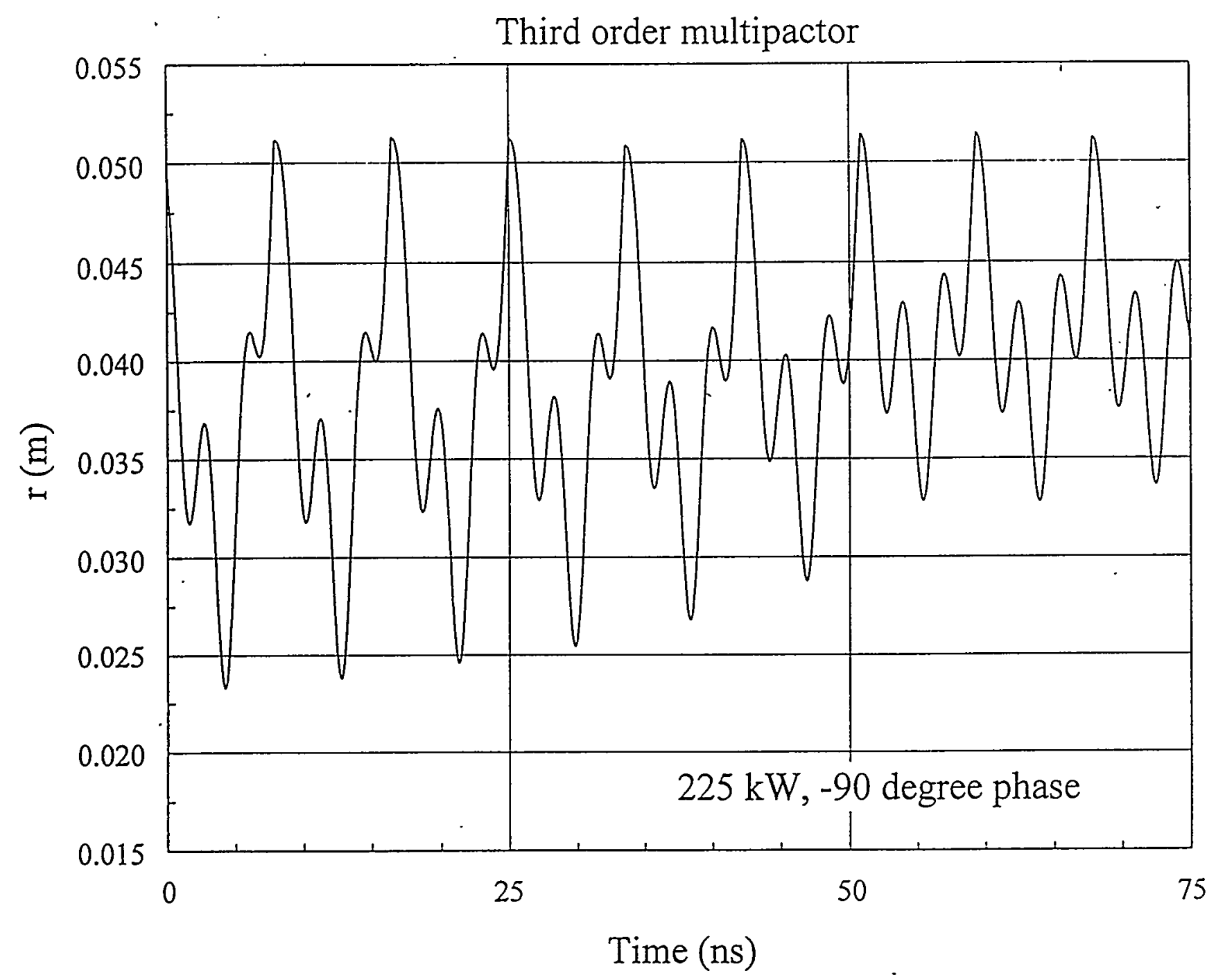

Figure 9. Electron orbit, $r(t)$ in the $75 \Omega$ line at $225 \mathrm{~kW}$. Initial emission from the outer surface at $-90^{\circ}$ phase. 


\section{APPENDIX 2 \\ WaveSim Control File}

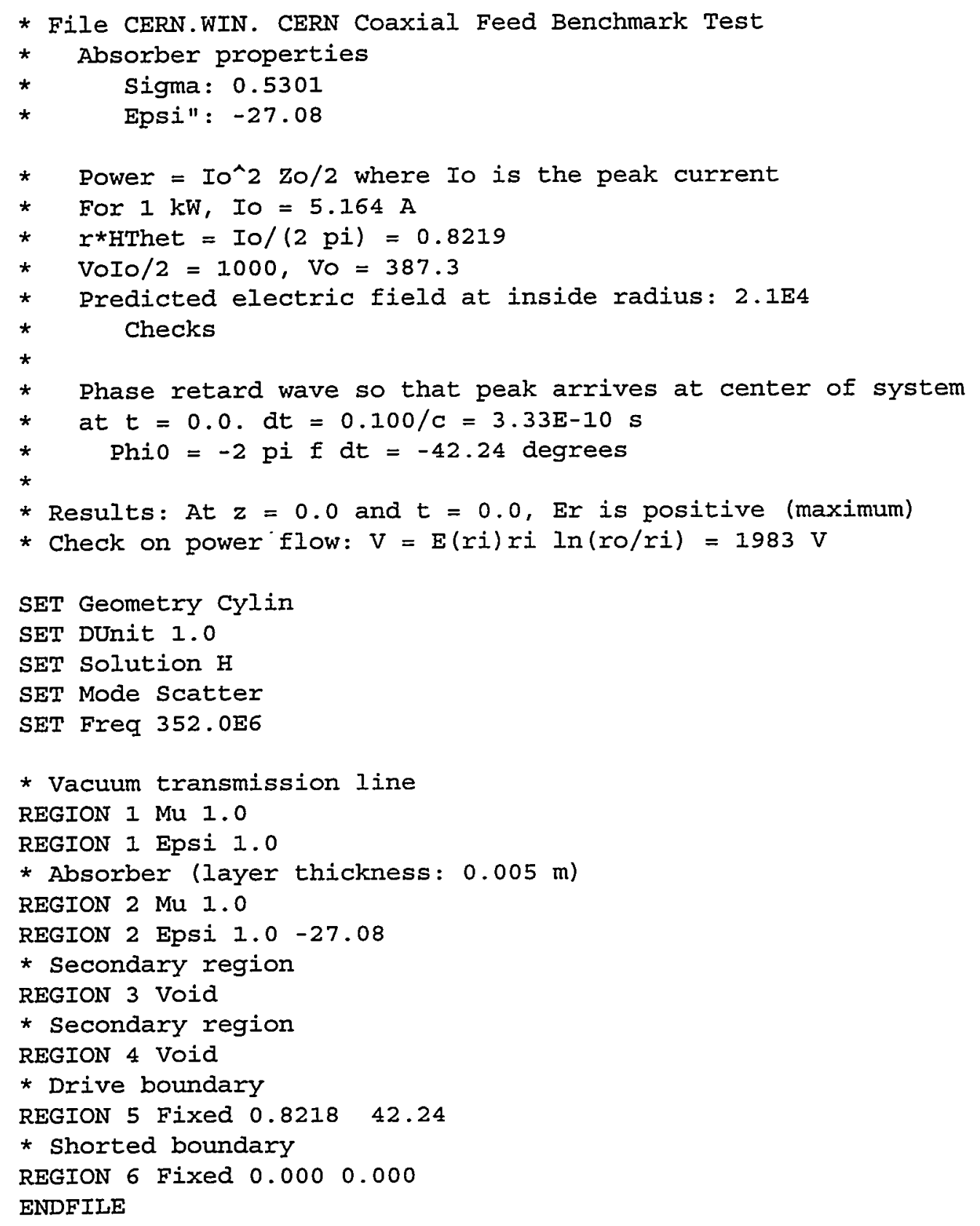




\section{APPENDIX 3 \\ Example of TRAK_RF Control File}

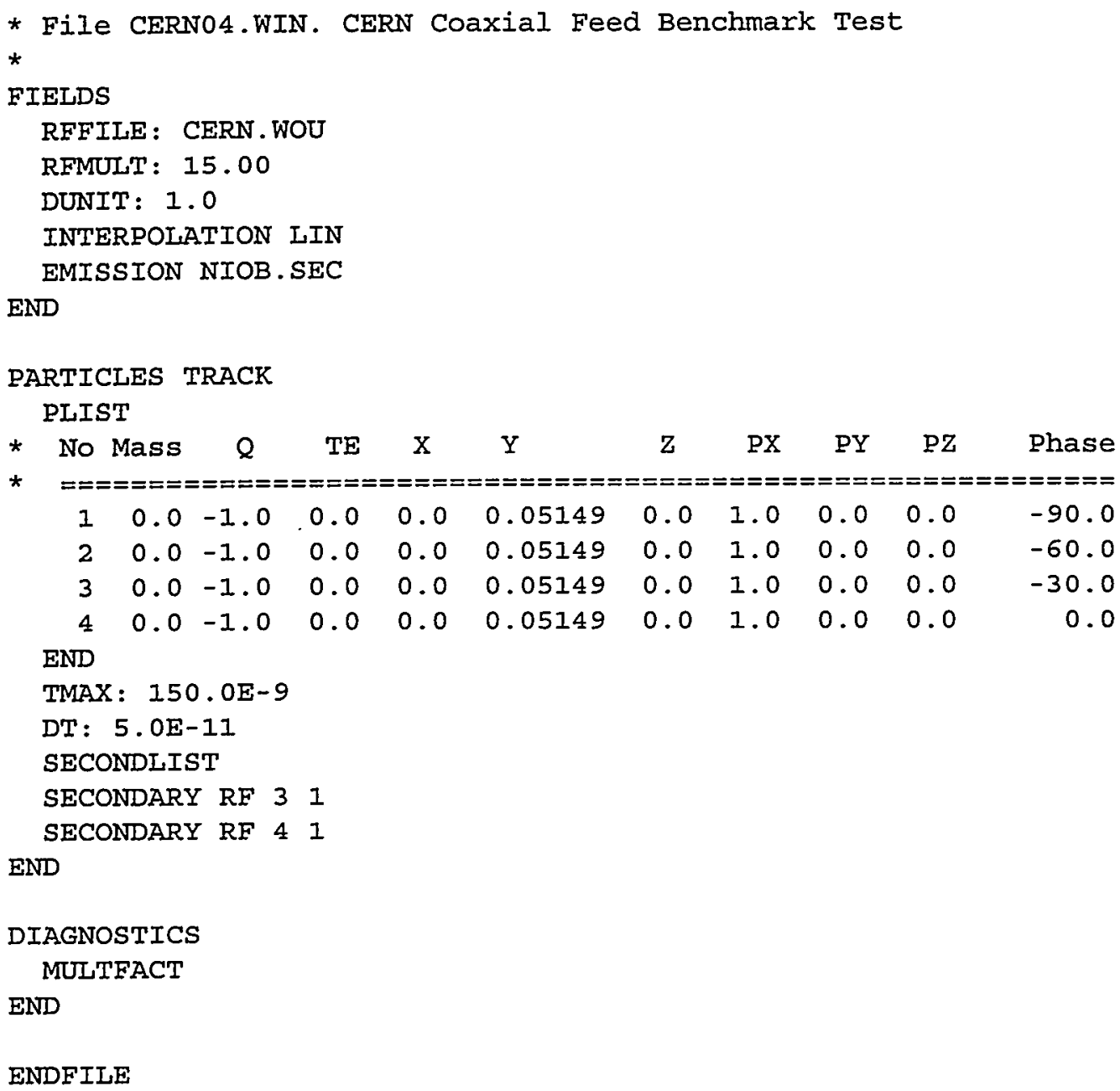




\section{APPENDIX 1 \\ Mesh File for the RF Solution}

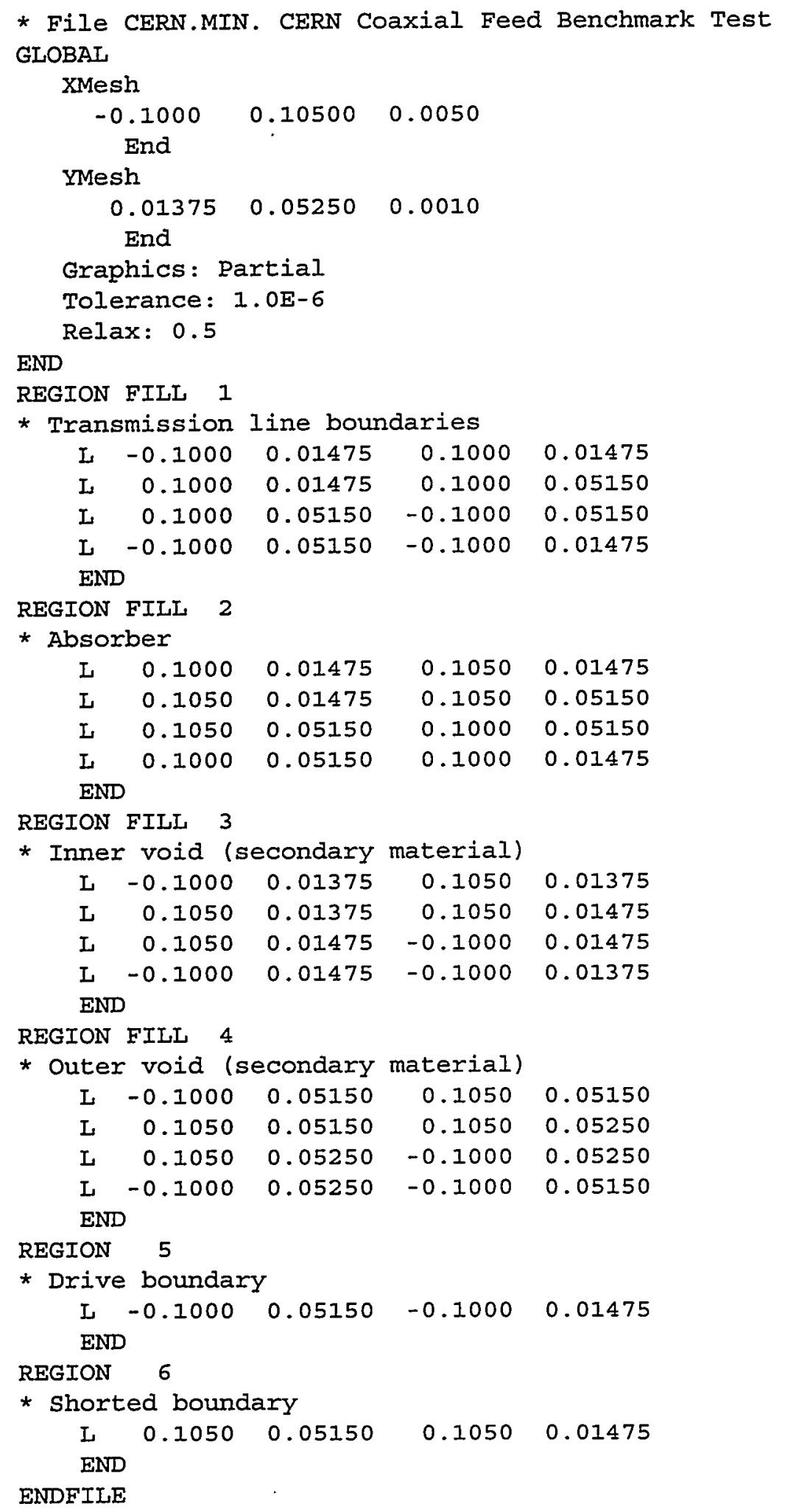


Trak RF uses the standard mesh generator and static field solvers of the TriComp system ${ }^{4}$. This suite of finite-element programs runs on IBM standard personal computers under DOS, Windows 3.1 or Windows 95. Boundary information is entered through an interactive drafting program or from popular CAD software like Autocad. The basic electrostatic and magnetostatic programs EStat and BStat use the linear finite-element formulation of Ref. 5 with solutions by successive over-relaxation. The solvers analyze files of boundary and material information to produce output files of vertex coordinates and corresponding values of electrostatic or vector potential. Figure 2 shows an example, a magnetostatic field generated by a cylindrical coil. Note the use of variable mesh resolution for an efficient approximation of infinite space boundary conditions. There are several other quasi-static solvers in the TriComp series that create solution files compatible with Trak_RF. Two advanced electrostatic solvers were developed for medical applications of RF fields: EStatA handles non-linear and anisotropic dielectrics while PAC calculates harmonic field penetration into imperfect dielectrics. For magnetic fields, the PerMag program treats complex anisotropic materials, include permanerit magnets with non-linear demagnetization curves. The Pulse program handles diffusion of magnetic fields created by pulsed coils with arbitrary current waveform and Nelson calculates harmonic field penetration into materials with eddy currents.
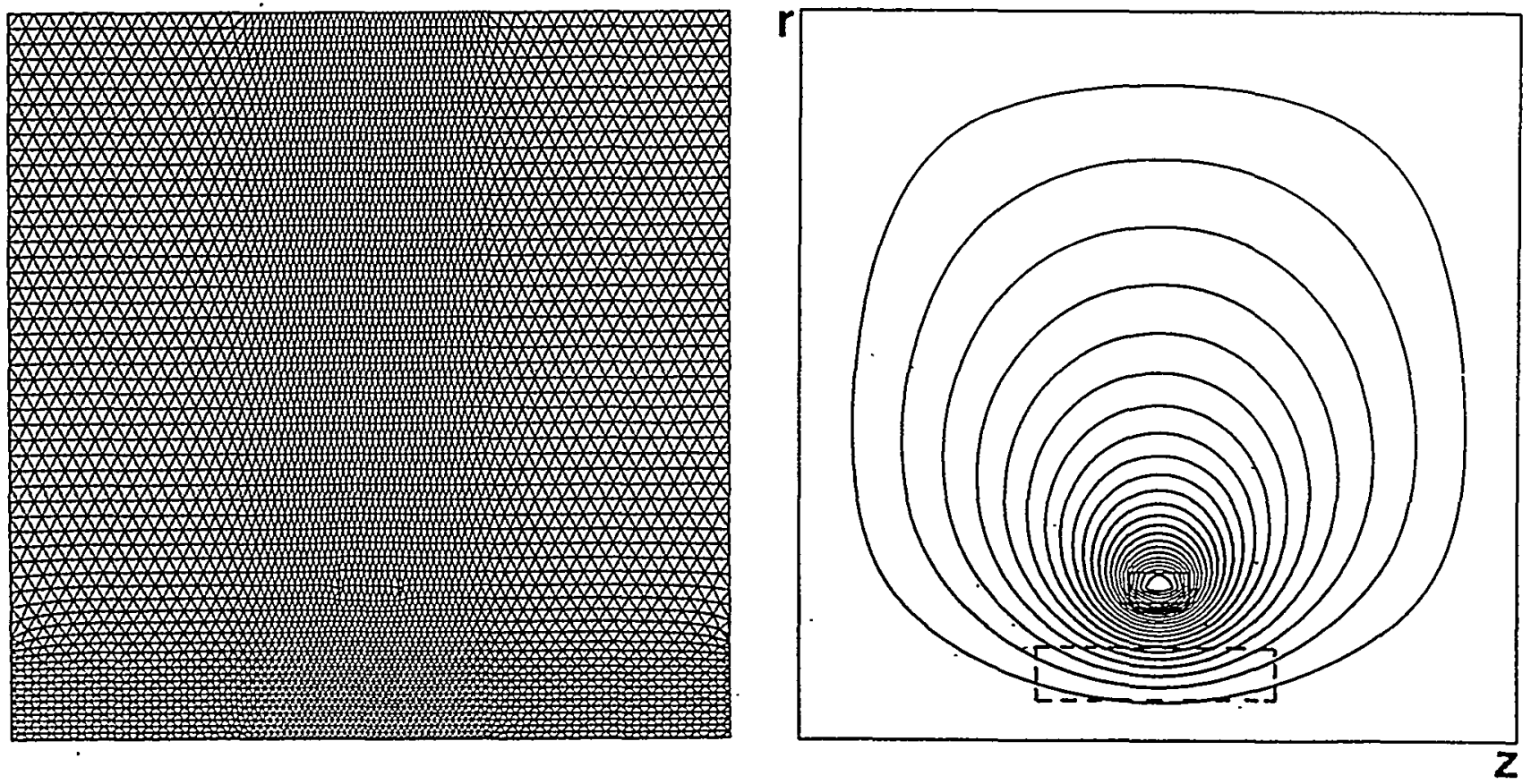

Figure 2. Field lines of a magnetostatic solution. Field from a single coil carrying current 2640 A. $r_{\min }: 0.0^{\prime \prime}, r_{\max }: 24.0^{\prime \prime}, z_{\min }:-12.0^{\prime \prime}, z_{\max }: 12.0^{\prime \prime}$. Dashed line shows approximate, extent for the RF solution of Fig. 1. 


\section{RF field computations}

The derivation of finite-element equations for two-dimensional frequency domain $\mathrm{RF}$ fields is reviewed in Ref. 6 . As an example, consider the equations for a planar structure with no variation in $z$ with electric field polarization $\mathrm{E}_{z}$. The associated differential equation is

$$
-\nabla \times\left(\frac{1}{\mu} \nabla \times E\right)=-\epsilon \omega^{2} E+j \omega J_{0} .
$$

The quantities $\mu$ and $\epsilon$ in Eq. 1 may have may have complex values to represent losses from resistivity or non-ideal materials. The current source $J_{0}$ contains information on the amplitude and phase of drive regions. Figure 3 shows the geometry and indices of vertices and elements surrounding a test vertex. In the example, the mesh has a regular logic with six elements encompassing each vertex. The finite-

element equation for wave propagation at the test vertex are determined by taking area integrals of Eq. 1 over the surface surrounded by the dashed line in Fig. 3 . The result is

$$
E_{z 0}\left(-\sum_{i} W_{i}+\omega^{2} \sum_{i} \frac{\epsilon_{i} a_{i}}{3}\right)+\sum_{i} E_{z i}+=j \omega \sum_{i} \frac{J_{t} a_{i}}{3} .
$$

In general, the quantities $E_{z \mathrm{i}}$ are complex numbers to represent amplitude and phase. The index $i$ refers to the vertices surrounding the test vertex marked $o$. The quantities $\epsilon_{i}, \mu_{i}$ and $J_{i}$ are the material properties and current density of the elements shown in Fig. 3. The geometric coefficients are given by

$$
W_{i}=\frac{\cot \theta_{b i+1} / \mu_{i+1}+\cot \theta_{a l} / \mu_{i}}{2},
$$

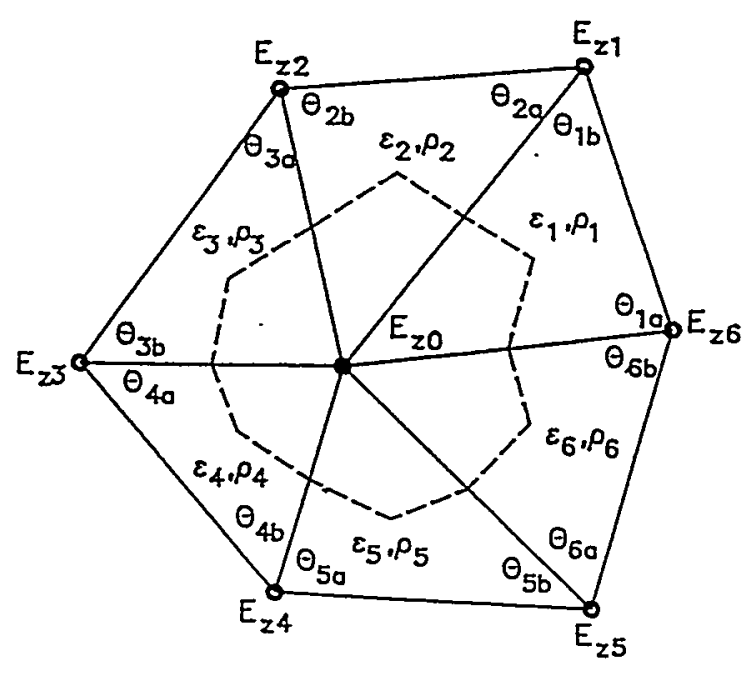

Figure 3. Geometry and labeling conventions for elements and vertices adjacent to a vertex of a finite element mesh with regular logic. 
Equation 1 represents a large set of coupled linear equations, one for each mesh vertex. The set is solved in Trak_RF using matrix inversion with block decomposition methods. The complex values of $E_{z i}$ gives the physical electric field at a given $R F$ phase. Numerical derivatives give the magnetic field components $B_{x}$ and $B_{y}$. For resonant type solutions in closed regions, we can use the current sources as coupling loops to create RF fields. The most convenient way to initiate fields in scattering solutions is with a drive boundary with a fixed complex value of $E_{z}$. In solutions with $E_{z}$ polarization, a metal wall is represented by the fixed value $E_{z}=[0,0]$. The open circuit condition is the natural boundary of finite-element solutions. For waves with $\mathrm{H}_{2}$ polarization, the Neumann condition represents a metal wall. A significant advantage of the finite-element method is the ability to define absorbing layers of arbitrary shape to represent free-space boundary conditions. The procedure is to set up a thin layer of width $\delta$ on the outside of the solution volume. The imaginary part of the dielectric constant in the layer is assigned the value $\epsilon "=-\sigma / \omega$, where the conductivity is matched to the impedance of the adjacent medium,

$$
\sqrt{\frac{\mu}{\epsilon}}=\frac{1}{\sigma \delta} .
$$

The performance of absorbing layers equals or exceeds that of look-back techniques ${ }^{7}$. The advantages are that termination layers can have any shape or orientation and do not place restrictions on the time step in time-domain solutions. Furthermore, layers can be constructed with generalized reactive and resistive impedance components to represent imperfect matches to downstream devices.

To illustrate the method, Fig. 4 shows a benchmark test of a high power coaxial feedthrough using the mesh of Fig. 1. In the cylindrical geometry the finite-element equations are solved for $\mathrm{H}_{\theta}$ to derive TEM type waves. The boundary on the left is a drive surface with the fixed stream function value $\mathrm{rH}_{\theta}$ $=[18.79,0]$ to produce $700 \mathrm{~kW}$ of electromagnetic energy flux. The region on the right is an absorbing layer of thickness $0.1^{\prime \prime}(0.00254 \mathrm{~m})$. The choice of material properties $\mu=\mu_{\mathrm{o}}$ and $\epsilon=\epsilon_{\mathrm{o}}(1-53.62)$ gives a termination matched to the impedance of free space. In time-domain tests, reflected energy from the termination is less than 1 part in $10^{5}$. Figure 4 shows contours of $\mathrm{rH}_{\theta}$ which lie along electric field lines. The magnitude of the electric field is proportional to the distance between lines divided by the relative dielectric constant. Note the accumulation of lines inside the alumina window with $\epsilon_{\mathrm{r}}=$ 7.8 .

\section{Charged-particle orbit calculations}

Charged-particle orbit calculations in Trak_RF are straightforward. They involve Runge-Kutta integrations using numerically-calculated field components. The main challenge is organization of the broad range of possibilities. The program can handle three numerical field solutions on independent conformal meshes: electrostatic, magnetostatic, and electromagnetic. The motivation for this versatility is to model techniques for stray electron control near RF windows such as sweeping fields. 

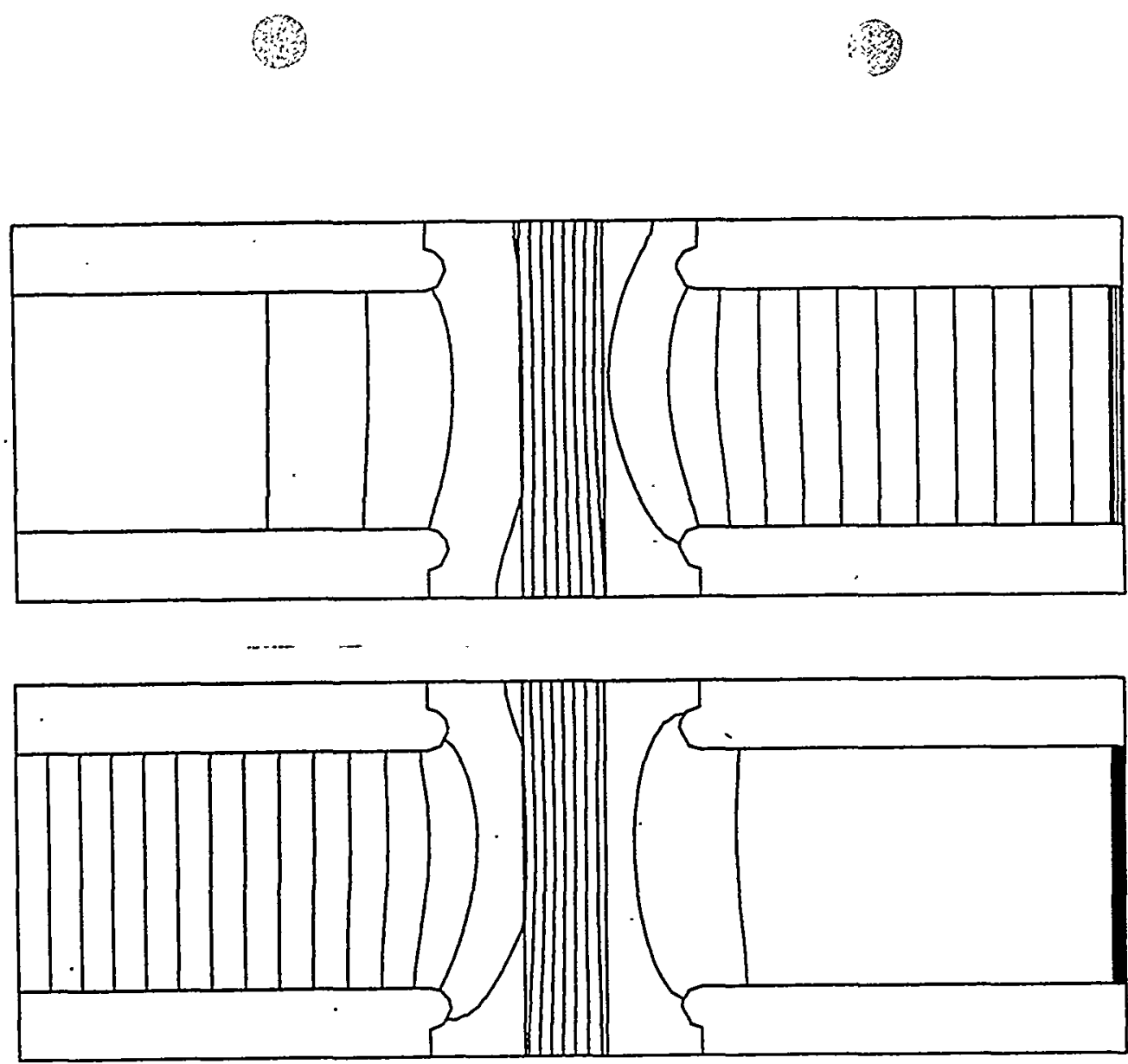

Figure 4. Field solution for the geometry of Fig. 1 at $350 \mathrm{MHz}$. Contours of $\mathrm{rH}_{\theta}$ are parallel to electric field lines. a) Real part of $\mathrm{rH}_{\theta}$. b) Imaginary part of $r \mathrm{H}_{\theta}$.

Field geometries can be mixed in any combination. The quasi-static solutions may have either rectangular geometry (variations in $\mathrm{x}$ and $\mathrm{y}$ with infinite extent in $\mathrm{z}$ ) or cylindrical (variations in $\mathrm{r}$ and $z$ with azimuthal symmetry). There are four possibilities for electromagnetic fields: rectangular geometries with primary field components $\mathrm{E}_{\mathrm{z}}$ or $\mathrm{H}_{2}$ or cylindrical systems with solutions for $\mathrm{E}_{\theta}$ or $\mathrm{H}_{\theta}$. Trak_RF uses a reference three-dimensional Cartesian coordinate system and organizes interpolations of the numerical field solutions to derive total values of $\mathbf{E}$ and $\mathbf{B}$ at the position and elapsed time of the particle. The three field solutions can be assigned translations and rotations within the threedimensional reference system.

The basic method to initiate particle orbits is through a parameter listing file generated by spreadsheets or user-written programs. The file specifies charge, mass, initial kinetic energy, position and direction cosines. When electromagnetic fields are present there is the option to assign reference phase. Each particle is initially assigned a multiplication factor of unity. Trak_RF can handle up to 1000 orbits in a run. The program also has the capability to generate a variety of particle distributions. Working from user-supplied numerical tables, Trak_RF can create arbitrary distributions in energy, position, and direction. The program has several options to stop orbits, including maximum distance and elapsed time. It is also possible to set up stopping planes along the Cartesian axes for high-accuracy interpolations of crossing particle parameters. An important feature for the multipactor application is stopping when a particle enters a material element. Element 
characteristics are identified by the status of the corresponding mesh region. Regions can be individually set to one of three conditions: Vacuum, Material or Secondary. A particle stops if it enters a Material element on any of the three field meshes. For Secondary elements, the orbit is returned to its position before entering the surface and assigned a low momentum in the opposite direction. The particle multiplication factor is multiplied by the secondary emission coefficient of the material. This quantity is either a constant value or derived from a user-generated numerical function of the incident kinetic energy. Orbits that reach the end of their lifetime with multiplication factors much larger than unity indicate the possibility of multipactoring.

\section{E. Benchmark calculation}

The following example demonstrates the capabilities of Trak_RF. We shall consider electron orbits near the vacuum window of the system of Fig. 1 for a power level $700 \mathrm{~kW}$. The solution incorporates the static solenoidal magnetic field of Fig. 2. The field is collinear with the transmission line with a maximum at the axial position of the window to repel electrons accelerated by the RF fields. To generate interesting orbits, the peak magnetic field value was set to 0.0125 tesla, the condition for electron cyclotron resonance at $f=350 \mathrm{MHZ}$. Figure 5 shows resulting orbits on the vacuum side projected in the reference $x-y$ plane along with the inner and outer boundaries of the downstream . transmission line. The increase of electron kinetic energy is clearly visible. There is also a simultaneous acceleration in $z$ away from the window. Ultimately, the electrons strike the walls several inches from the window.

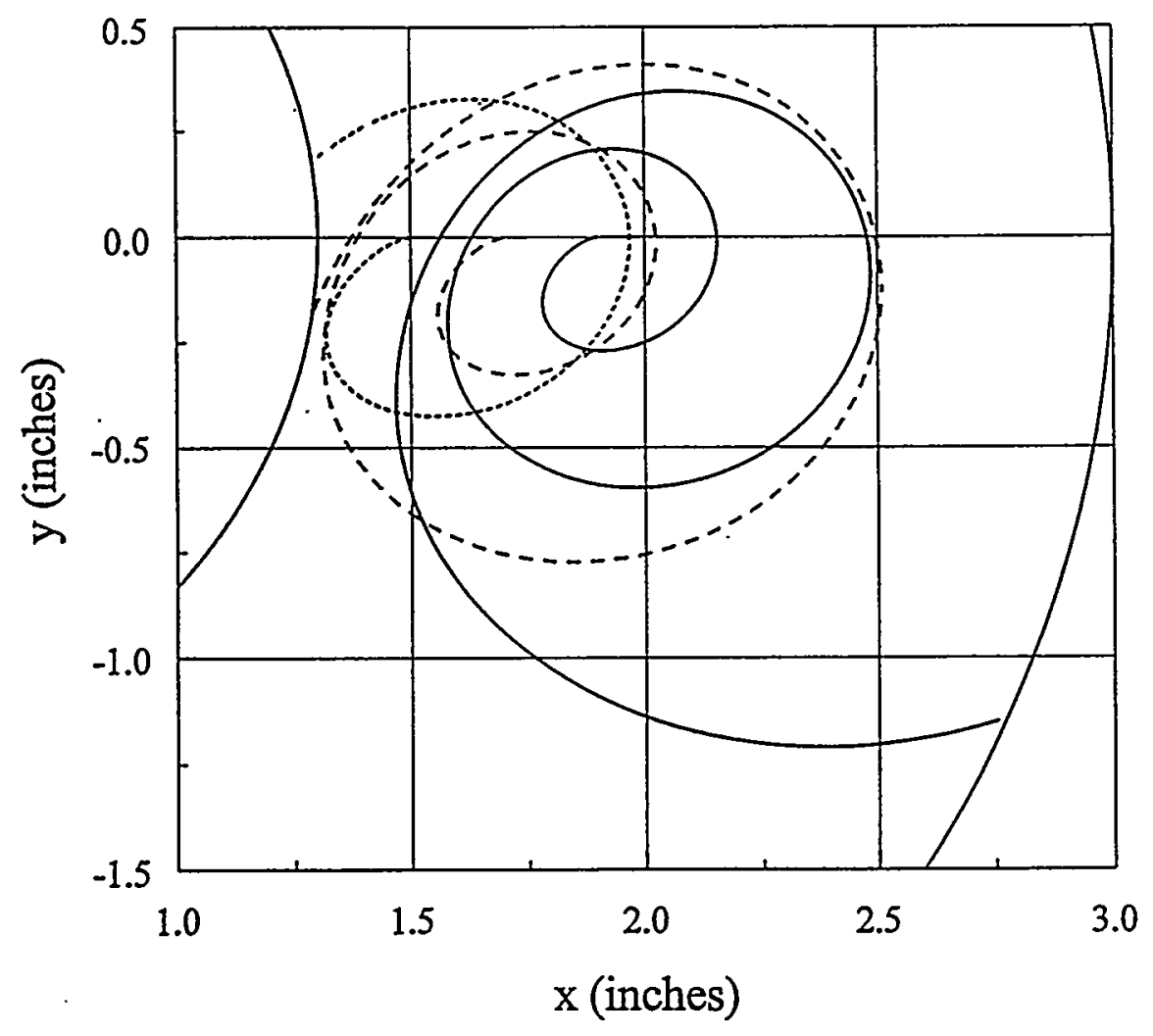

Figure 5. Electron orbits in combined fields of Fig. 3 and 4 - projection in the $x-y$ plane. 


\section{References}

1. S. Humphries, Jr., J. Comp. Phys. 125, 488 (1996).

2. G. Lawrence, et.al., Conventional and Superconducting RF Linac Design for the APT Project in Proc. 1996 Int'l. Linear Acc. Conf. (Geneva, 1996), to be published.

3. D. Rees, P.J. Tallerico and M. Lynch, IEEE Trans. Plasma Sci. 25, 1033 (1996).

4. TriComp mesh generator and solution programs courtesy of Field Precision Company, Albuquerque, New Mexico.

5. See for instance, J.L. Warren, et.al. Reference Manual for the Poisson/Superfish Group of Codes (Los Alamos National Laboratory, LA-UR-87-126, 1987), unpublished.

6. S. Humphries, Jr., Field Solutions on Computers (CRC Press, Boca Raton, 1977), Chap. 14. 7. See, for instance, K.S. Kunz and R.J. Luebbers, The Finite Difference Time-Domain Method for Electromagnetics (CRC Press, Boca Raton, 1993), Chap. 18. 


\title{
WaveSim \\ Frequency-domain Finite-element Electromagnetic Field Solutions
}

\author{
Instructions
}

Stanley Humphries, Jr.

Department of Electrical and Computer Engineering

University of New Mexico

Albuquerque, New Mexico 87131

\section{Program function}

WaveSim performs a wide variety of frequency-domain electromagnetic field calculations. The program derives two-dimensional solutions by finite-element methods on flexible triangular meshes. The present version handles planar and cylindrical geometries. Planar solutions have field variations in $\mathrm{x}$ and $\mathrm{y}$ with no variation along $\mathrm{z}$. In cylindrical solutions variations are in $\mathrm{r}$ and $\mathrm{z}$ with azimuthal symmetry.

WaveSim gives fast solutions using sparse matrix inversion techniques. The program covers the full range of electromagnetic calculations.

- Scans to investigate the frequency response of bounded systems

- Automatic searches to locate resonances of close ideal and lossy structures

- Scattering solutions at given frequency for open and lossy systems, including free-space conditions 
WaveSim applies a unique method of matched termination boundary layers in scattering calculations. These layers effectively represent open-space boundaries. They are more versatile and efficient than the look-back techniques used in finite-difference calculations. The method of distributed sources is another novel feature of WaveSim. It allows you to generate ideal incident plane waves in a bounded anechoic chamber for studies of electromagnetic wave scattering.

The material model of WaveSim is flexible. You can specify real and imaginary parts of the dielectric constant and magnetic permeability at the target frequency for up to 127 material regions. Ideal resistive materials can also be defined for frequency scans and absorbing layers in free-space solutions. An interactive graphical post-processor gives plots and scans of primary and secondary fields with automatic labeling of the mode options. The program automatically calculates volume and surface integrals to determine material and wall losses in microwave devices.

\section{Section 1. Installing and running WaveSim}

The TriComp Mesh program must already be in place to install WaveSim. Insert the distribution disk into a floppy drive and log to the drive. Type

INSTALI <ENTER >

The Install batch file checks that the TriComp directory is set up and copies the WaveSim programs to the directory $\mathrm{C}: \backslash$ TriComp and the example files to $\mathrm{C}: \backslash$ TriComplExamples.

A Wavesim run consists of three parts handled by three programs: mesh generation, field solution, and analysis. The programs communicate through data files. Sometimes, you may make several solutions from the same mesh by changing the characteristics of materials. Before beginning, add \TriComp to the DOS Path statement. The programs MESH.EXE, WSIM.EXE, DOSXMSF.EXE and WSV.EXE must be in a directory on the current DOS path. Each run includes the following steps.

- Prepare a Mesh Input Command File with a name of the form FName.MmN following the instructions in the Mesh Instruction Manual. Here, FName is any valid DOS file name.

- Run MESH by entering

Mesh FName

to create the mesh data file FName.MOU.

- Prepare a WaveSim Input Command File with a name of the form FName.WIN following 
the instructions in this manual.

- With both FName.MOU and FName.WIN in the current directory, run Wavesim by entering

WaveSim FName

The program performs the matrix inversion and creates a file FName.WOU.

- Analyze the solution using the WSV program. Run WSV and load FName or another solution file.

\section{Section 2. Structure of the WaveSim command file}

As with all the TriComp programs, the input file for WaveSim is an ASCII text file with commands and parameters. The file must end with the EndFile command. The file has a name of the form FName.WIN, where FName is the same as the prefix for the Mesh data file. The entries on a line can be separated by the following delimiters

Space, Comma, Forward slash, Colon, Tab, Equal sign

Any number of delimiters can be used in a line. Blank lines and comment lines are ignored. Comment lines begin with '*' (asterisk-space). With the exception of region numbers, all parameters are real numbers. The following formats are valid.

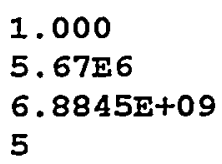

The last number is interpreted as 5.0 .

The program will accept commands in any order. The following example illustrates a complete control file.

* Wavesim file: CORRUG.WIN

* Transition from a corrugated structure to a cylindrical

SET Geometry Cylin 


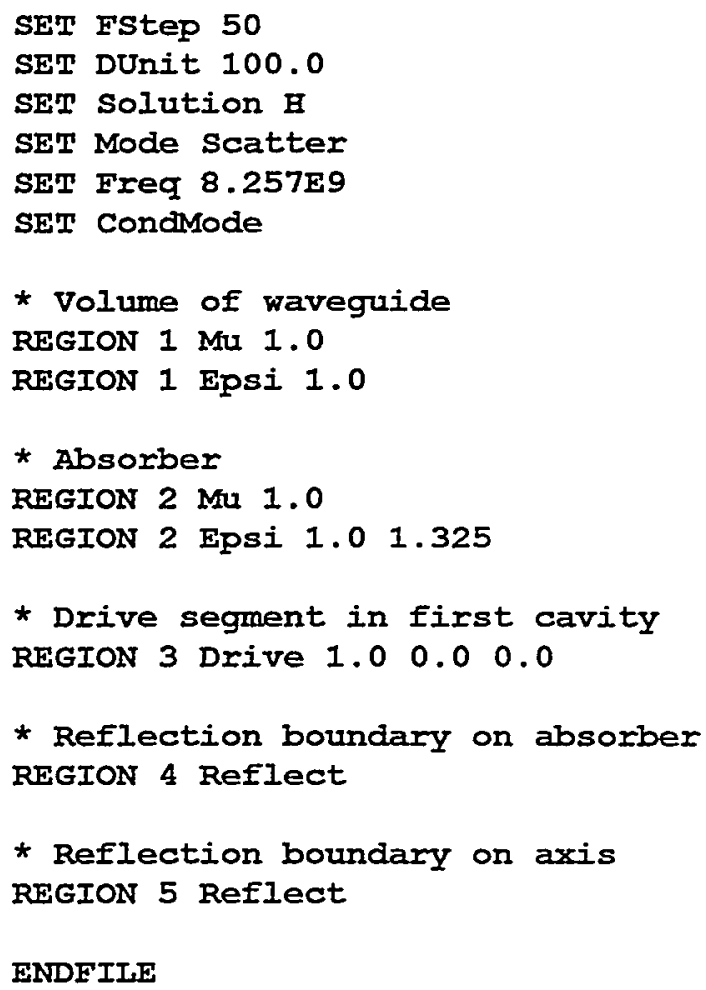

\section{Section 3. Commands for basic program control}

WaveSim commands divide into two groups: those that control the program operation and those that set the physical properties of regions. We begin with the program commands. They begin with the keyword SET. Each command is written as it might appear in the FName. WIN file.

\section{SET SOLUTION H}

The keyword Solution is used to set the primary field quantity following the choices for two-dimensional solutions listed in Table 1. The parameters are the characters $\mathbf{E}$ or $\mathbf{H}$ in either upper or lower case. In $\mathrm{E}$ wave solutions the primary field quantity is either $\mathrm{E}_{\mathrm{z}}$ or $\mathrm{rE}_{0}$. In $\mathrm{H}$ wave solutions the primary field quantity is either $\mathrm{H}_{2}$ or $\mathrm{rH}_{\theta}$. 


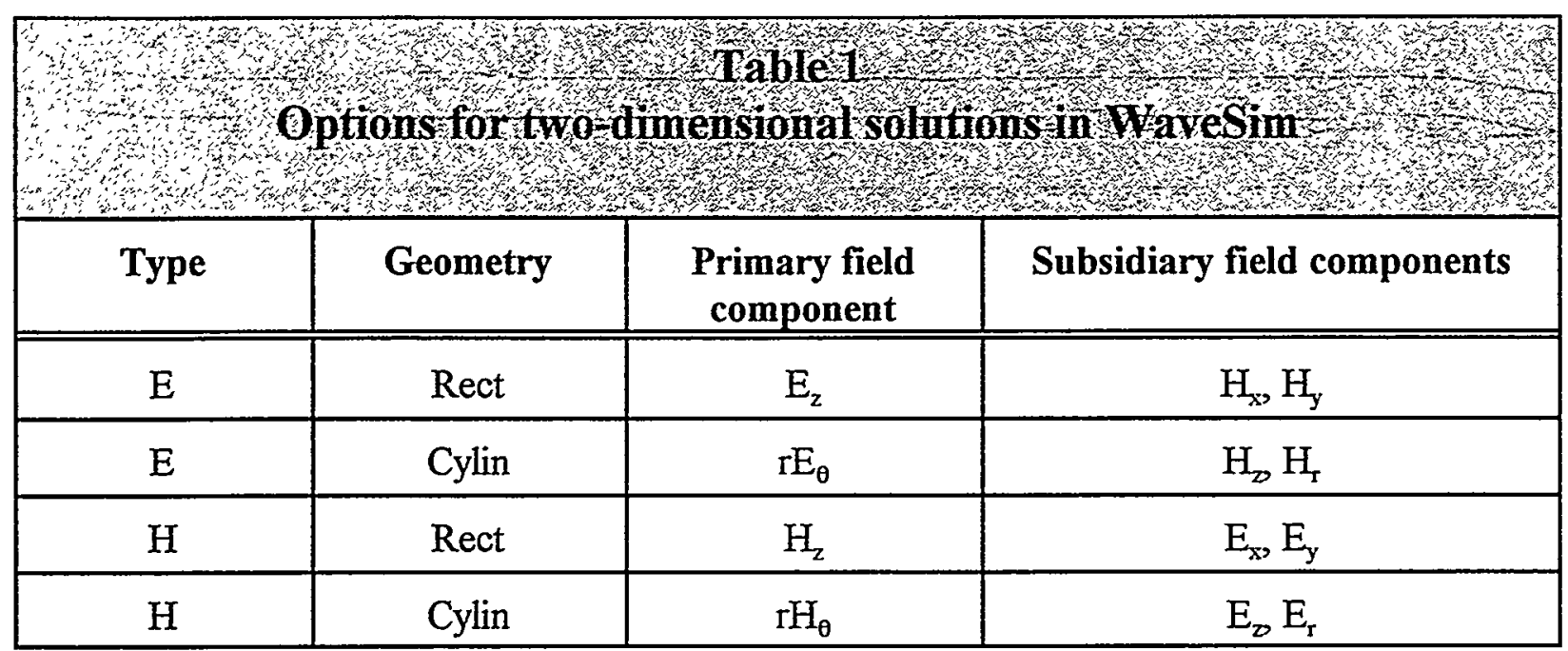

\section{SET GEOMETRY CYLIN}

The keyword Geometry sets the solution symmetry according to the choices of Table 1.

The parameter options are the strings RECT or CYLIN. All units and interpolation methods are automatically adjusted in WSim and WSV.

\begin{tabular}{|l|l|l|l|}
\hline \multicolumn{1}{|c|}{ Function } & \multicolumn{1}{|c|}{ Comments } \\
\hline \hline Scatter & $\begin{array}{l}\text { Simulation of open or closed } \\
\text { systems with specified frequency } \\
\text { and drives }\end{array}$ & $\begin{array}{l}\text { Single solution at the given frequency } \\
\text { output to a data file FName. WOU. }\end{array}$ \\
\hline Scan & $\begin{array}{l}\text { Searches for resonances in } \\
\text { closed structures or peak output } \\
\text { from open structures }\end{array}$ & $\begin{array}{l}\text { A sequence of solutions with a probe } \\
\text { listing of the primary field component at a } \\
\text { given location. No data file. }\end{array}$ \\
\hline Search & $\begin{array}{l}\text { Search for a resonant mode } \\
\text { within a frequency range for } \\
\text { ideal or lossy structures }\end{array}$ & $\begin{array}{l}\text { Several solutions to search for maximum } \\
\text { probe response followed by output to a } \\
\text { data file FName.WOU at the final } \\
\text { frequency. }\end{array}$ \\
\hline
\end{tabular}




\section{SET MODE SEARCH}

The keyword MODE sets the nature of the calculation according to the options of Table 2 . The parameter options are the strings Scatter, Scan or Search. Scan and Search calculations involve several solutions and may lead to extended run rimes.

\section{SET DUNIT 100.0}

The keyword DUnit signals the specification of length parameters in units other than meters. The real number parameter is the number of input units per meter. For example, with $D_{\text {unit }}=100.0$ enter all lengths in $\mathrm{cm}$. Be sure that the dimensions of regions in the Mesh input file are specified in the same units.

\section{SET FREQ 100.0E6}

The keyword FREQ sets a value for the wave frequency ( $(\mathrm{f})$ in $\mathrm{Hz}$ for Scatter type calculations. This command has no effect in the Search and Scan modes.

\section{SET RANGE 120.0E6 150.0E6}

The keyword RANGE defines a range of frequencies for Scan or Search calculations. The parameters are $f_{\min }$ and $f_{\max }$ in $\mathrm{Hz}$. In the Scan mode, WaveSim performs a series of calculations from $f_{\min }$ to $f_{\max }$ in uniform frequency steps of $\left(f_{\min }-f_{\max }\right) / f_{\text {step }}$ (see the FStep command below). In the Search mode, $f_{\min }$ and $f_{\max }$ define a frequency interval for the search that should bracket a single resonance. The program will not proceed unless than probe response changes sign over the interval. The command has no effect in the Scatter mode.

\section{SET FSTEP 25}

The keyword FSTEP sets the number of frequency steps in the Scan mode or the maximum of cycles in the Search mode. The integer parameter is the number of steps. The defaults values are 20 in the Sean mode and 7 in the Search mode. The command has no effect in the Scatter mode.

\section{SET TOLERANCE 5.0E-5}

The keyword TOLERANCE sets the target accuracy for a resonance search in the Search mode. The real number parameter is the target accuracy $\epsilon$. WaveSim exits the search when the relative change in frequency between two cycles satisfies $\Delta f / f<\epsilon$. The default value is $1.0 \times 10^{-4}$. The command has no effect in the Scatter and Scan modes. 


\section{SET PROBE 0.010 .02}

The keyword PROBE sets a location for a virtual probe that senses the real and imaginary parts of the primary field component for the Scan and Search modes. The real number parameters are the approximate coordinates of the position, either $(x, y)$ or $(r, z)$. WaveSim places the probe at the nearest vertex. The positions should be entered in meters or alternate units if the DUnit command appears in the input file. This command has no effect in the scatter mode.

\section{SET CONDMODE}

The standard input method for lossy materials is to give relative values for the real and imaginary parts of the dielectric constant $\left(\epsilon^{\prime} / \epsilon_{0}, \epsilon^{\prime \prime} / \epsilon_{0}\right)$ and the magnetic permeability $\left(\mu^{\prime} / \mu_{0}, \mu^{\prime \prime} / \mu_{0}\right)$. In the Scan and Search modes it is sometimes better to keep the effective material conductivity constant over the frequency range. This is true when we want to maintain an ideal absorbing layer. This command signals WaveSim to expect the quantities $\omega \epsilon^{\prime \prime} / \epsilon_{\mathrm{o}}$ or $\omega \mu " / \mu_{\mathrm{o}}$ as the imaginary material properties. In this case, the program calculates $\epsilon^{\prime \prime} / \epsilon_{\mathrm{o}}$ and $\mu^{\prime \prime} / \mu_{\mathrm{o}}$ for each frequency by dividing the quantities by $\omega$. For an ideal resistive material, the dielectric quantity equals $\omega \epsilon^{\prime \prime} / \epsilon_{\mathrm{o}}=\sigma / \epsilon_{\mathrm{o}}$, where $\sigma$ is the conductivity is mhos $/ \mathrm{m}$.

\section{SET LOWQ}

For a resonance search in an ideal or high-Q structure, WaveSim looks for a zero in the reciprocal of either the real or imaginary part of the probe response. In low $Q$ structures it is sometimes better to search for a zero of the direct probe signal. This command activates the low $Q$ search option.

\section{SET PLANEWAVE 13.6 0.0 5000.0 T}

This command sets up an incident plane electromagnetic wave for scattering solutions in Rectangular geometry using the method of virtual sources. There are three real number parameters.

$\mathrm{k}_{\mathrm{x}}$ : The wave number in $\mathrm{m}^{-1}$ along the $\mathrm{x}$ direction

$\mathrm{k}_{\mathrm{y}}$ : The wave number in $\mathrm{m}^{-1}$ along the $\mathrm{y}$ direction

A: The wave amplitude for the primary field component

Depending on the mode, the quantity A may have units of $\mathrm{V} / \mathrm{m}, \mathrm{V}, \mathrm{A} / \mathrm{m}$ or $\mathrm{A}$. As an example, the primary field for an $\mathrm{E}$ wave solution in planar geometry field has the variation 


$$
E_{z}\left(x_{2} y, t\right)=A \exp \left[j\left(k_{x} x+k_{y} y-\omega t\right)\right]
$$

Values of $k_{x}>0$ and $k_{y}=0$ give a plane wave propagating in the pesitive $x$ direction. The optional single-character parameter $T$ controls the field solution output. If it appears, WaveSim makes a data file of the total field - incident plus scattered. Otherwise, the programs records the scattered field only. For more information on free-space scattering calculations, see the NREPLACE command.

\section{Section 4. Commands to set material properties}

Because frequency-domain solutions are meaningful only for linear materials, the set of WaveSim commands to define region material properties is simple. The commands to set the physical properties of materials all start with the word Region and have similar format:

Region RegNo Reyword value [s]

Here, the integer RegNo is the Region Number defined in the Mesh input file. Keyword is one of the options listed below and Value[s] represents one or more parameters.

\section{REGION 4 REFLECT}

The keyword REFLECT designates that primary field has the fixed value $[0.0,0.0]$ in the region. The reflection condition represents a metal surface (short-circuit boundary) for $E$ type waves and an ideal open non-radiating boundary (open-circuit) for $\mathrm{H}$ type waves.

\section{REGION 7 VOID}

A VOID boundary is the inverse of a REFLECT boundary. It represents a metal wall for $\mathrm{H}$ type waves and an open-circuit for $E$ type waves. WaveSim implements internal void regions by setting $\epsilon=\epsilon_{\mathrm{o}}$ and $\mu=10^{12} \mu_{\mathrm{o}}$ for $\mathrm{E}$ type waves and $\epsilon=10^{12} \epsilon_{\mathrm{o}}$ and $\mu=\mu_{\mathrm{o}}$ for $\mathrm{H}$ type waves. The WSV program suppresses field line plotting in void regions. Note that all unspecified external boundaries automatically assume VOID conditions.

\section{REGION 2 EPSI $5.8-56.03$}

This command sets the dielectric constant for elements of a region. The first real number parameter is the real part of the relative dielectric constant, $\epsilon^{\prime} / \epsilon_{\mathrm{o}}$. The optional second 
parameter equals the imaginary part of the dielectric constant, $\epsilon " / \epsilon_{o}$, if Condhiode is not set. Otherwise, enter the quantity $\omega \epsilon " / \epsilon_{\mathrm{o}}$. (See the SET CONDMODE command.) The dielectric constant assumes a real value if there is no second parameter. The default value for all regions is $[1.0,0.0]$.

\section{REGION 2 MU $5.8-56.03$}

This command sets the magnetic permeability for elements of a region. The first real number parameter is the real part of the relative magnetic permeability, $\mu^{\prime} / \mu_{\mathrm{o}}$. The optional second parameter equals the imaginary part of the magnetic permeability, $\mu^{\prime \prime} / \mu_{\mathrm{o}}$ if CondMode is not set. Otherwise, enter the quantity $\omega \mu " / \mu_{\mathrm{o}}$. (See the SET CONDHODE command.) The magnetic permeability assumes a real value if there is no second parameter. The default value for all regions is $[1.0,0.0]$.

\section{REGION 7 FIXED 2500.045 .0}

The keyword FIXED indicates a fixed field region that acts as a wave source. The two real parameters are the amplitude and phase of the primary field at all points in the region. Specify the amplitude in the standard units of the primary field and the phase in degrees.

\section{REGION 3 DRIVE $4500.0-90.0 \quad 0.0$}

The keyword DRIVE indicates that the region has a drive current density. The first real number is the amplitude of the drive. The interpretation of the number and units depends on the geometry and solution type. Table 3 shows the options. The second real number parameter is the phase in degrees. The third optional parameter gives the direction of the current for $\mathrm{H}$ wave initiation. The quantity is the angle of the current density vector (in degrees) in the $x-y$ plane relative to the $x$-axis for planar problems. For cylindrical problems, the quantity gives the angle in the $\mathrm{r}-\mathrm{z}$ plane relative to the $\mathrm{z}$ axis. The parameter has no meaning for $E$ wave solutions where the current density points along the direction of the primary field $(z$ or $\theta$ ). Note that you can specify values of relative dielectric constant and magnetic permeability in a drive region using the EPSI and MU commands. 


\begin{tabular}{|c|c|c|c|}
\hline \multicolumn{3}{c}{} & \\
\hline & & & \\
\hline & & & \\
\hline
\end{tabular}

\section{REGION 5 NREPLACE 2}

This command is used to implement the distributed source method for free-space scattering solutions. The source terms are computed as through the scattering object were removed from a homogenous region of space. In this command, the first integer specifies the number of a region that constitutes a part of the scattering object. The second integer is the region number of the bulk of the solution volume. This volume carries a plane wave in the absence of the scattering object. Each region of the scattering object must have an NREPLACE command. (See also the SET PLANEWAVE command.)

\section{Section 5. Ideal absorbing boundaries}

Boundaries that absorb 100 per cent of the incident wave energy are used to terminate waveguides or the output ports of microwave devices. They are essential to simulate free-space conditions in scattering solutions. The method used in WaveSim is physically similar to resistive terminations on transmission lines. We define a thin layer and assign a resistive impedance matched to the characteristic impedance of the adjacent medium. The theory is described in Sects. 13.3 and 14.2 of the Appendices. This section gives a step-by-step summary of how to construct a termination layer boundary.

- During mesh generation, be sure to include an extra material layers of uniform thickness $\Delta$ along absorbing boundaries. The layer should be only a single-element thick as the absorption approaches 100 per cent as $\Delta$ approaches zero. The outer surface of the termination layer should have an unspecified (open-circuit) boundary. 
- Calculate the characteristic impedance of the non-absorbing medium adjacent to the termination layer, $Z_{1}=377.3\left(\mu_{1}{ }^{\prime} / \epsilon_{1}{ }^{3}\right)^{1 / 2}$, where $\mu_{1}{ }^{3}$ and $\epsilon_{1}{ }^{\prime}$ are the real parts of the relative magnetic permeability and dielectric constant.

- Assign the values shown in Table 4 for the relative dielectric constant and magnetic permeability in the termination layer.

Note that the method is very flexible. You can also create boundaries with capacitive or inductive properties.

\begin{tabular}{|c|c|c|c|c|c|}
\hline Type & CondMode & $\epsilon_{2}^{\prime}$ & $\epsilon_{2}^{\prime \prime}$ & $\underline{\mu}_{2}^{\prime}$ & $\underline{\mu}_{2} "$ \\
\hline E & Off & $\epsilon_{1}{ }^{\prime}$ & $-1 / \omega \Delta z Z_{1} \epsilon_{0}$ & $\mu_{1}^{\prime}$ & 0 \\
\hline $\mathrm{H}$ & Off & $\epsilon_{1}{ }^{\prime}$ & 0 & $\mu_{1}{ }^{\prime}$ & $-Z_{1} / \omega \Delta z \mu_{o}$ \\
\hline$E$ & On & $\epsilon_{1}^{\prime}$ & $-1 / \Delta z Z_{1} \epsilon_{o}$ & $\mu_{1}^{\prime}$ & 0 \\
\hline $\mathrm{H}$ & On & $\epsilon_{1}^{\prime}$ & 0 & $\mu_{1}^{\prime}$ & $-Z_{1} / \Delta z \mu_{o}$ \\
\hline
\end{tabular}

\section{Section 6. Resonance searches}

WaveSim will automatically locate resonances of high Q structures in the Search mode. When defining boundaries in Mesh, include a small region for a drive current. For $\mathrm{E}$ type solutions, the drive should be near the anticipated location of maximum $\mathbf{H}$. For $\mathrm{H}$ type solutions, the drive should be near the location of maximum $\mathbf{E}$ and point in the direction of the electric field. The drive current may have any amplitude, but must have zero phase. Place a probe at the anticipated location of the maximum primary field closest to the drive point. Enter a range of frequencies that brackets the resonance using the Range command. If you are unsure of the approximate frequency or if there are several nearby resonances, run a Scan over the frequency range. For $\mathrm{H}$ type waves, a resonance occurs when the real part of the probe response goes from a positive to negative value. For $\mathrm{E}$ type waves, the imaginary part of the probe signal makes a zero crossing at resonance. During the search, WaveSim seeks the zero crossing of the reciprocal of the 
appropriate component of the probe signal. Sometimes it may be preferable to use the signal values directly. For this case enter the Set LowQ command.

\title{
Section 7. Command summary
}

WaveSim uses SI units (MKS) for all quantities. The one exception is that you can enter a scaling factor for distances with the DUnit command. The example library contains an extensive set of run files developed for program testing and applications. See the file DIRECT.TXT for a listing of current examples.

Run times grow rapidly with the size of the mesh. The run time scales with $k_{\max }$ and $l_{\max }$ (the number of elements along the $\mathrm{x}$ and $\mathrm{y}$ or $\mathrm{z}$ and $\mathrm{r}$ axes) as follows. If

$$
\begin{gathered}
M=\min \left(k_{\max } l_{\max }\right), \\
N=\max \left(k_{\max } l_{\max }\right)
\end{gathered}
$$

then the number of operations for a single is solution is given by

$$
\text { Operations } \propto N M^{3} \text {. }
$$

The actual time depends on the speed of your computer and the number of operations in Scan and Search mode. WaveSim lists the quantity Operations $/ 10^{6}$ on the screen to give you an idea of the relative run time.

\section{Program Control}

\author{
Set Solution $\mathrm{H}$ \\ Set Geometry Cylin \\ Set Mode Search \\ Set Dunit 100.0 \\ Set Freq 150.0E6 \\ Set Range 120.0E6 150.0E6 \\ Set FStep 25 \\ Set Tolerance 5.0E-5
}


Set Probe 0.010 .02

Set CondMode

Set LowQ

Set PlaneWave 13.6 0.0 5000.0 T

\section{Material Properties}

\section{Region 4 Reflect}

Region 7 Void

Region 2 Epsi 5.8 -56.03

Region 6 Mu 5.8 -56.03

Region 7 Fixed 2500.045 .0

Region 3 Drive 4500.0 -90.0 0.0

Region 5 NReplace 2

\section{Section 8. Running WSV}

WSV (for WaveSim View) is an interactive graphical analysis program for output files from WaveSim. The program interpolates the primary field and calculates gradients to find subsidiary fields. It also performs automatic calculations of field energy and surface current integrals. WaveSim makes a record of all numerical results in a history file, FName.DAT. With its on-screen menu support, WSV is largely self-explanatory. This section gives a brief summary of operation.

Run the program from the directory that contains the data files, FName.EOU. WSV immediately asks for a data file prefix. The program cannot operate without a resident data file. After loading, WSV displays the main menu. Commands are arranged by function. You can choose the following selections with a mouse or by entering the key letters.

\section{Main Menu}




\section{Get file}

Load a different data file

\section{Mesh plot}

Plot the computational mesh in the current view window

\section{Contour plot}

Make a contour plot of the primary field component. Choose either the real part, imaginary part of the magnitude using the Contour Plot Type command in the advanced plot menu.

\section{Toggle hardcopy}

When hardcopy is ON, wSV makes a hardcopy plot to the device specified in the WSV.CFG file as well as a screen plot. This option works with mesh, contour and scan plots using the current view coordinates.

\section{Point}

Make a field calculation at a point. The coordinates can be entered by pointing on the plot screen with the mouse or by typing numbers. Enter the coordinates in the length units used in the Mesh command file. Values are listed on the screen and recorded in the history file.

\section{Scan along line}

Makes a series of field calculations along a scan line. The starting and ending coordinates of the line are entered with the mouse or keyboard. WSV makes a complete listing in the history file and plots a chosen field component on the screen or hardcopy device. Use the Set Scan command to determine the plotted field quantity.

\section{Matrix file}

Makes a data file consisting of interpolated field values on a specified rectangular grid. The annotated file is in ASCII format and can be transferred to spreadsheets or your own analysis programs. The routine uses the current interpolation method and the field component determined by the Set Scan Type command.

\section{Energy analysis}

WSV scans through the computational mesh to calculate stored electromagnetic energy in regions with non-zero volume. The program writes the global value and energy content organized by region in the history file. There is also a listing of time-averaged power dissipation for materials with imaginary components of the dielectric constant or magnetic permeability. You can get figures for energy in resolve energy into can get resolution The program also lists the following area integral for line regions, $\iint \mathrm{dA} \mathrm{H}_{1}^{2} / 2$. Multiplication of this quantity by the surface resistivity of the region gives power dissipation in the skin depth of metal walls. To control the output, you can define special dummy line regions to represent metal walls during mesh generation. These lines do not affect the solution if they 
are not assigned properties in WaveSim.

\section{Advanced Plot Menu}

Go to the advanced plot menu to change plot parameters and make wireframe and colored element plots

\section{Program Menu}

Go to the program menu to check on available files and change program parameters.

\section{Advanced Plot Menu}

\section{Contour plot type}

Sets the field quantity for contour plots. The command displays a menu of choices.

\section{Number of contours}

Change the number of contour lines

\section{Contour limits}

Over-ride the automatic selection of contour limits.

\section{Scan plot type}

Sets the file quantity for on-screen plots of scans and the quantity recorded by the Matrix File command. The command display a menu of choices.

\section{Number of scan points}

Increase or decrease the number of values calculated in a scan. The default is 50 . The maximum number is 200 .

\section{Plot view}

Zoom in on a portion of the solution space for plots. Use the mouse or keyboard entries to define a view box.

\section{Restore global view}

Zoom out to the full solution view.

\section{Set snap distance}

For coordinate input, the mouse moves in incremental distances called the snap distance. This command allows you to enter a convenient increment. 


\section{Boundary plot}

Plot the boundaries of solution regions.

\section{Element plot}

Makes a screen plot of elements color-coded by the value of the primary field component set by the Contour Plot Type command. It may be necessary to use the Plot View command if the plot is too dense. There is no direct hardcopy for this command. The plots can be saved using screen capture software.

\section{Wireframe plot}

Makes a three dimensional wireframe plot of primary field component chosen in the Contour Plot Type command. The characteristics are set by the Wireframe Parameter command.

\section{Wireframe parameters}

Sets parameters for the wire frame plot. Press ENTER to leave a parameter unchanged. Enter ThetaZ and ThetaX in degrees to rotate the drawing. DView (default 2.5) adjusts the perspective - increase the value to move farther away. Increase the value of Mag to magnify the drawing (the default value is 1.0 ). Shift the drawing horizontally or vertically using XDisp or YDisp. You can add an coordinate axis plotted in the $\mathrm{T}=0$ plane. NAxis adjusts the number of grid lines along the coordinate directions

\section{Program Control Menu}

\section{List files}

List files with the current suffix in the current directory

\section{List run names}

List all files with the suffix EOU

\section{Set file suffix}

Set a suffix for the List File command. The DOS wildcards '*' and '?' are allowed.

\section{Scale current file}

Multiply all values of the primary field by a constant. This is useful to scale resonant mode calculations to target field values for power and energy calculations.

\section{Toggle interpolation}

The calculations for point and scan output normally use an adaptive least squares fit method. Although it gives good accuracy, this method sometimes fails for unusual 
geometries (i.e., target point inside a narrow concavity). The alternative is a simple linear interpolation using potential values at the vertices of the enclosing triangle.

\section{Toggle mouse}

Turns off mouse support so that all coordinate entry is through the keyboard. Repeat the command to turn the mouse back

\section{Operating system}

Temporary exit to DOS to change directories or run a simple editor. Enter EXIT to return to WSV. 


\title{
Multipactor Calculations for the LANL APT Superconducting Linac Cavities
}

\author{
Stanley Humphries, Jr. \\ Department of Electrical and Computer Engineering \\ University of New Mexico \\ Albuquerque, NM 87131
}

April 23, 1997

I made extended studies with TRAK_RF to investigate conditions for electron multipactoring in the superconducting cavities designed for the LANL APT accelerator. These are blind runs in the sense that I have no information about theoretically-predicted or experimentally measured results. I would like your reactions because I plan to include these studies in the paper I will submit to the Proceedings of the Particle Accelerator Conference.

I used the $700 \mathrm{MHZ}$ single-cell test geometries supplied by Frank Krawczyk at $\beta=0.64$ and 0.82 . Figure 1 shows the geometry of the $\beta=0.82$ cell. The region marked $A$ is a secondary emission region with the properties of niobium described in my previous report. Any electron striking elements in this region is re-emitted with the appropriate energy-dependent secondary emission coefficient. Region $B$ is vacuum and Region $C$ is a small drive current to generate the resonant mode. I added a multiplication factor to renormalize the field. The independent variable in the runs is the on-axis value of $E_{z}$ at the midpoint of the cavity.

Electrons were emitted during the acceleration half-cycle from the twelve equally-spaced locations shown in Fig. 1. There were 19 particles created at each position with phases from $-180.0^{\circ}$ to $0.0^{\circ}$ in $10^{\circ}$ intervals. Each run tracked a total of 228 electron orbits for $150 \mathrm{~ns}$ (105 $\mathrm{RF}$ cycles). Each electron started with a multiplication factor of unity. The factor either increased or decreased, depending on whether the electron struck a niobium surface with an energy in the range with $\delta>1.0$. A multiplication factor of 1.0 means either that particles were at 
the wrong RF phase in their first collision or that they left the solution region without striking a niobium surface.

Generally, multiplication factors less than unity imply that the cavity is not subject to electron multipactoring. Individual multiplication factors were less than unity and there was no orbit that stayed in resonance for a large number of cycles. Although the particles started at discrete emission sites, they wandered about during their lifetime, sampling different parts of the cavity. Therefore, I believe the results give some confidence that multipactoring will not be a problem in the low-field regime.

Figure 2 shows global multiplication factors as a function of cavity electric field. The figure plots the multiplication factor versus $\mathrm{E}_{\mathrm{z}}(0,0)$ in the range from 1 to $6 \mathrm{MV} / \mathrm{m}$ for the $\beta=$ 0.64 and 0.82 cavities. The $\beta=0.82$ cavity was safe below $4.2 \mathrm{MV} / \mathrm{m}$, but exhibited strong multipactor effects at higher gradient. Growing orbits generally started near the outer wall of the radius of the $20 \mathrm{~cm}$ radius cavity. Here, the combination of electric field amplitude and short transit distance gave kinetic energy at collision close to the optimum value. Table 1 shows starting orbit parameters for particles that had more than 100 collisions for the $\beta=0.82$ cavity. Electron multiplication resulted mainly from first-order multipactoring where electrons struck a surface each half cycle. Multiplication factors in Fig. 2 at high energy range were in the range of $10^{11}$ to $10^{14}$. For comparison, extending the peak multiplication factor to 100 collisions gives $1.3^{100}=2.5$ $\times 10^{11}$. Multipactoring began at a lower field value of $3.6 \mathrm{MV} / \mathrm{m}$ in the $\beta=0.64$ cavity.

In summary, the orbits look physically reasonable and the multiplication factor technique seems to provide an unambiguous indication of potential trouble. The resonance behavior is much stronger than I observed in the coaxial simulations, leading to large multiplication factors. 


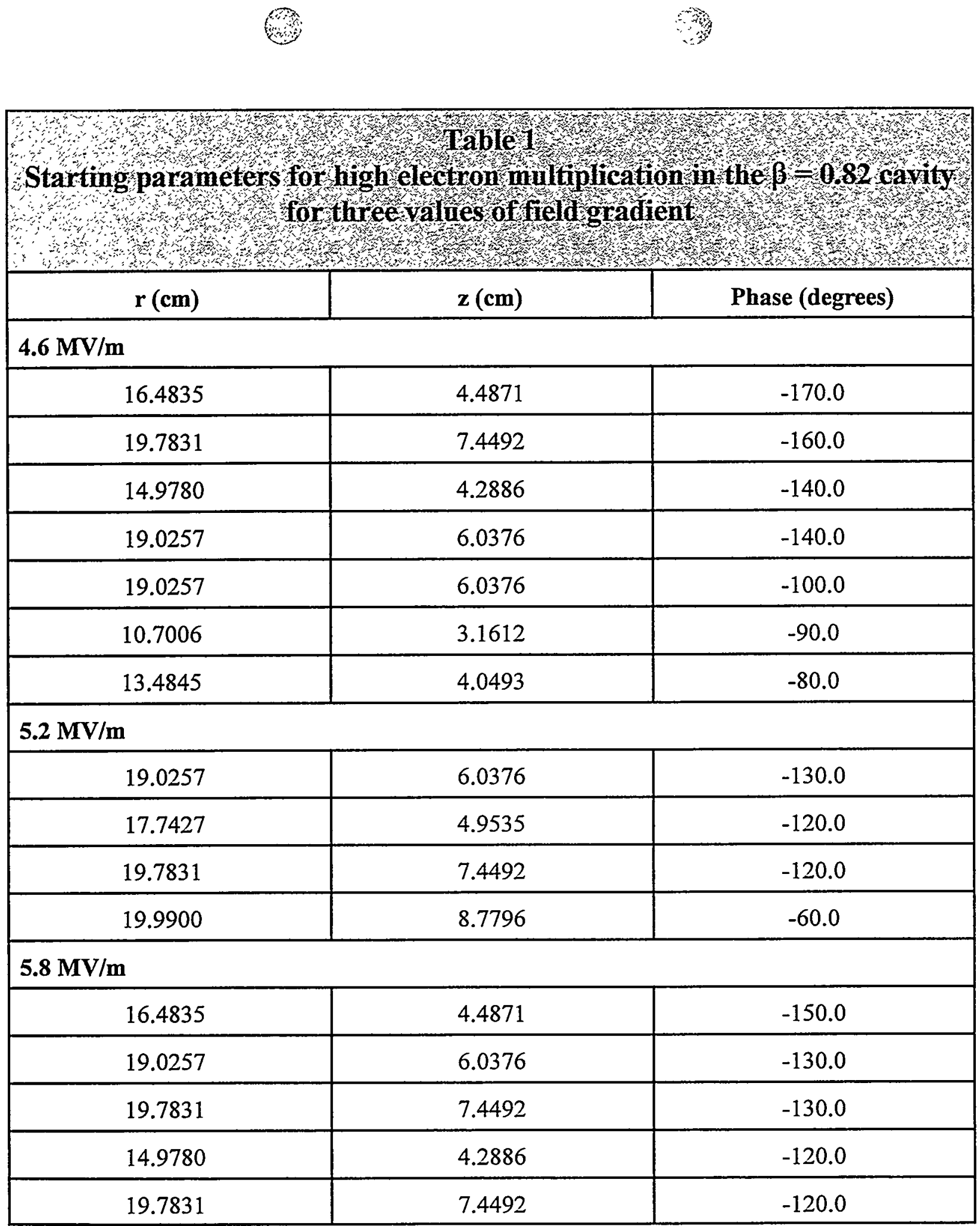




\title{
ELECTRON MULTIF CTOR CODE FOR HIGH-PO
}

\author{
S. Humphries, Jr., Department of Electrical and Computer Engineering, \\ University of New Mexico, Albuquerque, NM 87131 and \\ D. Rees, APT Program, Los Alamos National Laboratory, Los Alamos, NM 87545
}

\begin{abstract}
We have developed an integrated software system, TrakRF, to model electromagnetic fields and charged particle orbits in high-power RF devices. Our primary application is simulation of electron multipactoring near $R F$ vacuum windows for the APT (Accelerator Production of Tritium) Program. In this paper we describe features of the codes and initial multipactor calculations for superconducting cavity arrays. The finite-element frequency-domain solver can determine resonant modes in cavities and waveguides. TrakRF directly determines power dissipation and phase shifts in lossy materials. In addition, the program handles scattered waves in open structures with free-space boundaries based on matched termination layers. TrakRF has advanced particle tracking capabilities to investigate a variety of innovative window designs. The program can simultaneously apply three independent solutions for electrostatic, magnetostatic and RF fields. The finiteelement methods allow an accurate representation of electron collisions with surfaces.
\end{abstract}

\section{INTRODUCTION}

TrakRF is an integrated finite-element software system to model charged particle trajectories in combined electrostatic, magnetostatic and electromagnetic fields. The program is an extension of the Trak 3.0 [1] gun design code. New capabilities include frequency-domain calculations of electromagnetic fields in both resonant cavities and open spaces and particle tracking in timedependent fields. $A$ unique feature is the ability to combine up to three independent numerical solutions for static electric and magnetic fields and RF modes. The present version handles two-dimensional fields in planar or cylindrical geometries.

The software was developed to investigate contributions of multipactoring to breakdowns on RF windows in high-power accelerators. A cooperative program on Accelerator Production of Tritium to maintain the US nuclear weapons stockpile has recently been initiated at Los Alamos National Laboratory (LANL) and the Savannah River Site [2]. The goal is a continuously operating proton linac that produces a $1.3 \mathrm{GeV}$ beam with an average current of $100 \mathrm{~mA}$ (130 MW beam power). The accelerator demands powerful $R F$ systems with high reliability. A critical area of concern is the possibility of breakdowns on
$R F$ vacuum windows that must transmit $M W$ power levels. To address the issue, an experimental and theoretical program of window testing and development has been initiated at LANL [3]. Starting in October 1996, the University of New Mexico has supported this effort through the development of particle and radiation diagnostics to warn of impending breakdowns and computer codes to help understand the role of stray electrons in window failure.

\section{CONFORMAL MESHES AND STATIC FIELD COMPUTATIONS.}

All static and dynamic field calculations in TrakRF are carried out on conformal triangular meshes modeled on those used in SuperFish [4]. As an example, Fig. I shows a mesh of triangular elements for the calculation of resonant modes in the five cell $\beta=0.64$ superconducting cavities designed for the APT accelerator. The advantage is apparent - the edges of elements conform closely to curved and angled material boundaries. As a result, each element is uniquely associated with a material. This is an important feature in a particle tracking code because it allows an accurate identification of surface collisions. Section 3 reviews some advantages of the finite element formulation in RF calculations. In static field solutions, there are three major advantages over finite-difference calculations: 1) the finite element method gives accurate field values near metal surfaces, 2) the technique correctly represents field discontinuities at the boundaries of dielectrics and ferromagnetic materials, and 3) it is easy to implement Neumann conditions on angled and curved boundaries.

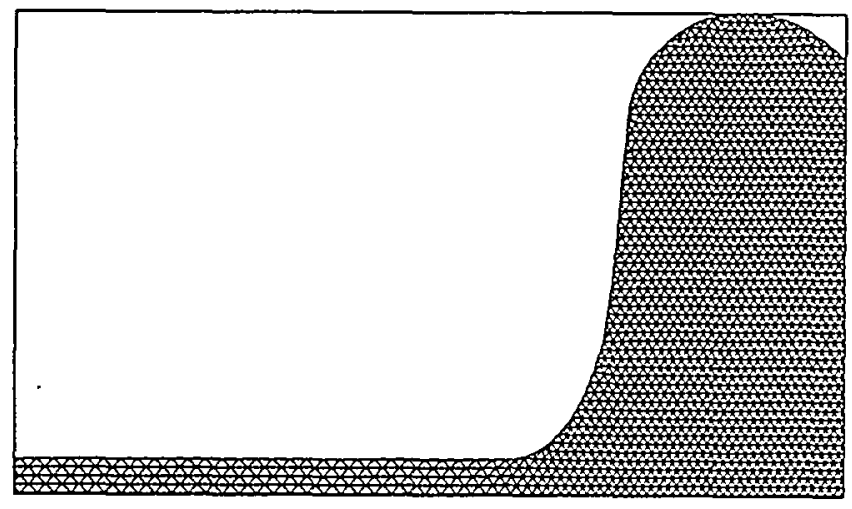

Figure 1. Conformal triangular mesh for the LANL 5 cel] superconducting cavity 
TrakRF uses the standard mesh gen 0 rond static field solvers of the TriComp system [5]. This suite of finiteelement programs runs on IBM-standard personal computers. Boundary information is entered through an interactive drafting program or from drafting like AutoCAD. The basic electrostatic and magnetostatic solvers use the linear finite-element formulation described in Refs. [6] and [7] with solutions by successive over-relaxation. They analyze files of boundary and material information to produce output files of vertex coordinates and corresponding values of electrostatic or vector potential.

\section{RF FIELD COMPUTATIONS}

The derivation of finite-element equations for twodimensional frequency-domain RF fields is reviewed in Ref. [7]. As an example, consider the equations for a planar structure with no variation in $z$ with electric field polarization $E_{z}$. The associated differential equation is

$$
-\nabla \times\left(\frac{1}{\mu} \nabla \times E\right)=-\epsilon \omega^{2} E+j \omega J_{0},
$$

where $\omega$ is the angular frequency of the radiation. The quantities $\mu$ and $\epsilon$ may have may have complex values to represent losses from resistivity or non-ideal materials. The current source $\mathrm{J}_{0}$ contains information on the amplitude and phase of drive regions. The finite-element equations for wave propagation at the test vertices are derived from area integrals of Eq. 1 over surrounding elements and vertices. The result is

$$
\begin{gathered}
E_{x 0}\left(-\sum_{t} W_{i}+\omega^{2} \sum_{t} \frac{\epsilon a_{i}}{3}\right)+\sum_{t} E_{z l}= \\
j \omega \sum_{t} \frac{J a_{i}}{3} .
\end{gathered}
$$

In general, the quantities $E_{z j}$ are complex numbers to represent amplitude and phase. The index $i$ refers to the vertices and elements surrounding a test vertex marked $o$. The quantities $\epsilon_{i}, \mu_{i}$ and $J_{i}$ are the material properties and current density of the elements. Expressions for the geometric coefficients $W_{i}$ are given in Ref. [7]. Equation 2 represents a large set of coupled linear equations, one for each mesh vertex. The set is solved in TrakRF using sparse matrix inversion methods. The complex values of $E_{z i}$ give the physical electric field at a given RF phase. Numerical derivatives give the magnetic field components $B_{x}$ and $B_{y}$.

A significant advantage of the finite-element method is the ability to define ideal absorbing layers of arbitrary shape to represent free-space boundary conditions. The procedure is to set up a thin layer of width $\delta$ on the outside of the solution volume. The imaginary part of the dielectric constant in the layer is as ${ }^{\prime}$ d the value $\epsilon^{\prime \prime}=-\sigma / \omega$, where the conductivity is matched to the impedance of the adjacent medium,

$$
\sqrt{\frac{\mu}{\epsilon}}=\frac{1}{\sigma \delta} .
$$

The performance of absorbing layers equals or exceeds that of look-back techniques [8]. The advantages are that termination layers can have any shape or orientation and do not place restrictions on the time step in time-domain solutions.

Figure 2 shows an example of a resonant calculation, the $\pi$ mode of the LANL five-cell superconducting cavity array for proton beams with $\beta=0.64$. The outer radius is $19.4 \mathrm{~cm}$ and there is a $20 \mathrm{~cm}$ beam pipe on the left that is not shown. A small capacitive probe (bottom-right) drives the mode. The program searches for the zero crossing of the imaginary part of the response of a sensor near the outer radius. The predicted frequency of $701.6 \mathrm{MHZ}$ is in good agreement with SuperFish and MAFIA results.

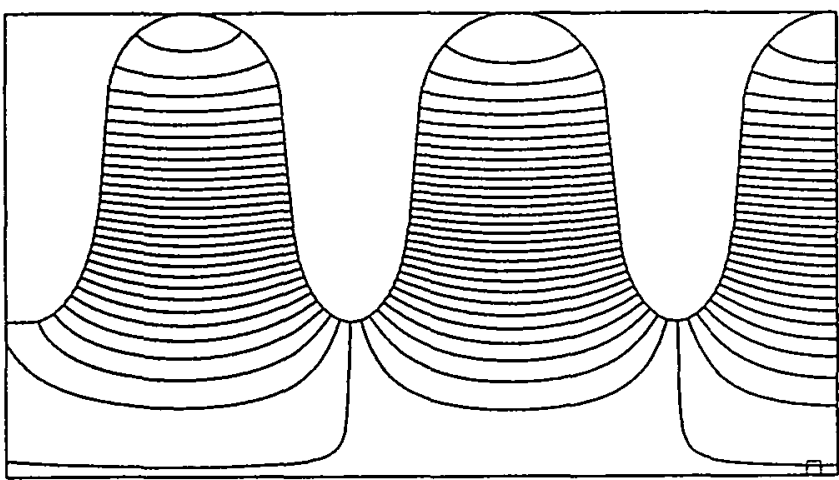

Figure 2. Electric field lines $-\pi$ mode of the LANL $\beta=$ 0.645 -cell superconducting cavity array. $f=701.6 \mathrm{MHZ}$.

\section{CHARGED-PARTICLE ORBIT CALCULATIONS}

Charged-particle orbit calculations in TrakRF are straightforward. They involve Runge-Kutta integrations using numerically-calculated field components. The main challenge is organization of the broad range of possibilities. The program can handle three numerical field solutions on independent conformal meshes: electrostatic, magnetostatic, and electromagnetic. The motivation for this versatility is to model stray electron control near RF windows using sweeping fields. Field geometries can be mixed in any combination. The static solutions may have either rectangular geometry (variations in $\mathrm{x}$ and $\mathrm{y}$ with infinite extent in $z$ ) or cylindrical (variations in $r$ and $z$ with azimuthal symmetry). There are four possibilities for electromagnetic fields: rectangular geometries with primary field components $\mathrm{E}_{\mathrm{z}}$ or $\mathrm{H}_{\mathrm{z}}$ or cylindrical systems with solutions for $E_{\theta}$ or $H_{\theta}$. TrakRF uses a reference 
three-dimensional Cartesian cook 3 ate system and organizes interpolations of the numerical field solutions to derive total values of $\mathbf{E}$ and $\mathbf{B}$ at the position and elapsed time of the particle. The three field solutions can be assigned translations and rotations within the threedimensional reference system.

The basic method to initiate particle orbits is through a parameter listing file generated by spreadsheets or userwritten programs. The file specifies charge, mass, initial kinetic energy, position and direction cosines. When electromagnetic fields are present there is the option to assign a reference phase. Each particle initially has a multiplication factor of unity. TrakRF can handle up to 1000 orbits in a run. The program can also generate a variety of particle distributions. Working from usersupplied numerical tables, TrakRF creates arbitrary distributions in energy, position, and direction. The program has several options to stop orbits, including maximum distance and elapsed time. It is also possible to set up stopping planes along the Cartesian axes for high-accuracy interpolations of crossing particle parameters.

An important feature for the multipactor application is orbit modification when a particle enters a material element. Element characteristics are identified by the status of the corresponding mesh region. Regions can be individually set to one of three conditions: Vacuum, Material or Secondary. A particle stops if it enters a Material element on any of the three field meshes. For Secondary elements, the orbit is returned to its position before entering the surface and assigned a low momentum in the opposite direction. The particle multiplication factor is multiplied by the secondary emission coefficient of the material. This quantity is either a constant value or derived from a user-generated numerical function of the incident kinetic energy. Orbits that reach the end of their lifetime with multiplication factors much larger than unity are susceptible to multipactoring.

\section{BENCHMARK CALCULATIONS}

We carried out an extensive set of runs to investigate multipactoring in the $\beta=0.64$ and $\beta=0.82$ single-cell test cavities at LANL. We used the energy-dependent secondary emission coefficients for clean niobium tabulated in Ref. [9]. Electrons were emitted during the acceleration halfcycle from twelve equally-spaced positions on the upstream wall. Nineteen electrons were generated at each position in the phase range from $-180^{\circ}$ to $0^{\circ}$ in $10^{\circ}$ intervals. Each run tracked a total of 228 electrons for $150 \mathrm{~ns}$ ( $105 \mathrm{RF}$ periods). The multiplication factor for individual electrons increased or decreased from unity depending on the history of interactions with the niobium walls. The global multiplication factor is the sum of individual values divided by the number of initial electrons. When the value was less than unity most individual factors were small and there was no orbit that stayed in resonance for a large number of cycles. Although partis started at discrete locations, resonant electrons migrated large distances; therefore, the runs gave a good indication of average cavity properties.

Figure 3 shows global multiplication factors as a function of the RF electric field amplitude at the center of the cavity over the range 1.0 to $6.0 \mathrm{MV} / \mathrm{m}$. The $\beta=0.82$ cavity was safe below about $4.2 \mathrm{MV} / \mathrm{m}$, but exhibited strong electron multiplication at higher gradient. Dangerous orbits generally started near the outer wall of the cavity. Here, the combination of electric field amplitude and transit distance gave resonant electrons that struck the walls with kinetic energy in the range $200-1000 \mathrm{eV}$. The increasing electron density resulted mainly from first order multipactoring, where critical electrons struck the cavity about once each half $R F$ cycle. Global multiplication factors as high as $10^{14}$ were observed for the 150 ns runs. The $\beta=0.64$ cavity had a lower critical field of about $3.6 \mathrm{MV} / \mathrm{m}$.

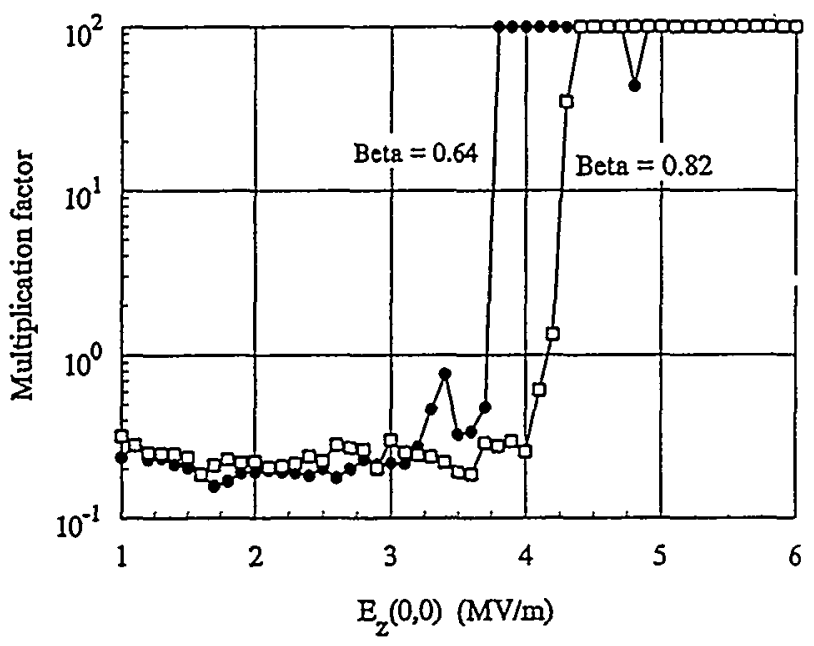

Figure 3. Global multiplication factor - electron multipactoring in the LANL single cell tests cavities as a function of peak on-axis field. The values at the top range from $10^{11}$ to $10^{14}$.

\section{REFERENCES}

1. S. Humphries, Jr., J. Comp. Phys. 125, 488 (1996).

2. G. Lawrence, et.al., Conventional and Superconducting $R F$ Linac Design for the APT Project in Proc. 1996 Int'l. Linear Acc. Conf. (Geneva, 1996), to be published.

3. D. Rees, P.J. Tallerico and M. Lynch, IEEE Trans. Plasma Sci. 25, 1033 (1996).

4. K. Halbach and R.F. Holsinger, Particle Acc. 7, 213 (1976).

5. TriComp software source code courtesy of Field Precision, Albuquerque, NM.

6. P.E. Allaire, Basies of the Finite Element Method (W.C. Brown Publishers, Dubuque, 1985).

7. S. Humphries, Jr., Field Solutions on Computers (CRC Press, Boca Raton, 1998), Chap. 14.

8. See, for instance, K.S. Kunz and R.J. Luebbers, The Finite Difference Time-D.omain Method for Electromagnetics (CRC Press, Boca Raton, 1993), Chap. 18.

9. D.R. Lide (ed..), Handbook of Chemistry and Physics, 74th Edition (CRC Press, Boca Raton, 1993), 12-107. 